\title{
A unified solution to the small scale problems of the $\Lambda$ CDM model
}

\author{
A. Del Popolo, ${ }^{a, b}$ J. A. S. Lima, ${ }^{c}$ Júlio C. Fabris, ${ }^{d}$ Davi C. \\ Rodrigues $^{d}$
}

${ }^{a}$ Dipartimento di Fisica e Astronomia, University Of Catania, Viale Andrea Doria 6, 95125 Catania, Italy

${ }^{b}$ International Institute of Physics, Universidade Federal do Rio Grande do Norte, Av. Odilon Gomes de Lima, 1722 - Capim Macio - 59078-400, Natal-RN, Brazil

${ }^{c}$ Departamento de Astronomia, Universidade de São Paulo, Rua do Matão 1226, 05508-900, São Paulo, SP, Brazil

${ }^{d}$ Departamento de Física, Universidade Federal do Espírito Santo, Av. Fernando Ferrari 514, Vitória - ES, Brazil

E-mail:

adelpopolo@oact.inaf.it;limajas@astro.iag.usp.br; fabris@pq.cnpq.br; davi.rodrigues@cosmo-ufes.org

\begin{abstract}
We study, by means of the model proposed in Del Popolo (2009), the effect of baryon physics on the small scale problems of the CDM model. We show that, using this model, the cusp/core problem, the missing satellite problem (MSP), the Too Big to Fail (TBTF) problem, and the angular momentum catastrophe can be reconciled with observations. Concerning the cusp/core problem, the interaction among dark matter (DM) and baryonic clumps of $1 \%$ the mass of the halo, through dynamical friction (DF), is able to flatten the inner cusp of the density profiles. We moreover assume that haloes form primarily through quiescent accretion, in agreement with the spherical collapse model (SCM)-secondary infall model (SIM) prescriptions. The results of this paper follow from the two assumptions above. Concerning the MSP and TBTF problem, applying to the Via Lactea II (VL2) subhaloes a series of corrections similar to those of Brooks et al. (2013), namely applying a Zolotov et al. (2012)-like correction obtained with our model, and further correcting for the UV heating and tidal stripping, we obtain that the number of massive, luminous satellites is in agreement with the number observed in the MW. The model also produces an angular momentum distribution in agreement with observations, that is with the distribution of the angular spin parameter and angular momentum of the dwarfs studied by van den Bosch, Burkert, \& Swaters (2001). In conclusion, the small scale problems of the CDM model can all be solved by introducing baryon physics.
\end{abstract}

Keywords: cosmology: theory - large scale structure of universe - galaxies: formation 


\section{Contents}

1 Introduction 1

2 Model 4

3 Results and discussion $\quad 9$

3.1 Cusp/core problem $\quad 10$

3.2 Angular momentum catastrophe 12

3.3 The Too Big to Fail and the Missing Satellites problems 17

4 Conclusions

\section{Introduction}

Despite the $\Lambda \mathrm{CDM}$ model being highly successful in describing the observations of the Universe, its large scale structure and evolution (Spergel et al. 2003, Komatsu et al. 2011; Del Popolo 2007, 2013, 2014), it has some problems in describing structures at small scales (e.g., Moore 1994; Moore et al. 1999; Ostriker \& Steinhardt 2003; Boylan-Kolchin, Bullock, and Kaplinghat 2011, 2012; Oh et al. 2011) ${ }^{1}$. The main problems are the a) cusp/core problem (Moore 1994; Flores \& Primak 1994), namely the discrepancy between the flat density profiles of dwarf galaxies and LSBs and the cuspy profile predicted by dissipationless N-body simulations (Navarro, Frenk \& White 1996, 1997; Navarro 2010); b) the "missing satellite problem" (MSP), namely the discrepancy between the number of predicted subhalos in Nbody simulations (Moore et al. 1999) and the one observed ${ }^{2}$; c) the angular momentum catastrophe (van den Bosch, Burkert,\& Swaters, 2001 (VBS)) namely the angular momentum loss in Smooth Particle Hydrodynamics ( $\mathrm{SPH}$ ) simulations of galaxy formation, giving rise to disks (of dwarf galaxies) having angular momentum distributions different than those of cold dark matter haloes, and disc size of simulated galaxies much smaller than the real ones.

Concerning the Cusp/Core problem, numerical simulations predict an inner cuspy profile, with inner slope $\rho \propto r^{\alpha}$, with $\alpha=-1$, from dwarf galaxies to clusters of galaxies, in the case of the Navarro, Frenk, \& White $(1996,1997)$ (hereafter NFW) profile, $\alpha=-1.5$ in the Moore et al (1998), and Fukushige \& Makino (2001) simulations, or different values according to the objects considered (Jing \& Suto 2000; Ricotti 2003; Ricotti \& Wilkinson, 2004; Ricotti Pontzen, \& Viel 2007; Del Popolo 2010 (DP10), Del Popolo 2011 (DP11); Cardone \& Del Popolo 2012; Del Popolo, Cardone, \& Belvedere 2013). More recently (Stadel et al. 2009; Navarro et al. 2010), it was shown that a better fit to density profiles is the Einasto profile, characterized by a profile that flattens (becomes shallower) going to the center, until values of $\alpha \simeq 0.8$ at $120 \mathrm{pc}$ (Stadel et al. 2009). The previously quoted smallest value for the slope obtained in simulations is larger than that in observations, which usually show flat or almost flat galaxy inner density profiles (Burkert 1995; de Blok, Bosma, \& McGauch 2003; Swaters et al. 2003; Del Popolo 2009 (DP09), Del Popolo 2012a,b (DP12a, DP12b); Del Popolo \&

\footnotetext{
${ }^{1}$ Other problems of the $\Lambda$ CDM model are the cosmological constant problem (Weinberg 1989; Astashenok, \&Del Popolo 2012), and the "cosmic coincidence problem".

${ }^{2}$ For the Milky Way (MW) the difference is larger than an order of magnitude.
} 
Hiotelis 2014; Oh et al. 2011; Oh et al. 2010, 2011; Kuzio de Naray \& Kaufmann 2011). The cusp/core problem, initially noticed in galaxies, is a problem also present in clusters of galaxies. The studies by Sand et al. (2004), and more recently those of Newman et al. (2009, 2011, 2013), found flat inner density profiles in the case of several clusters. Several solutions to the problem have been proposed (see de Blok 2010 for a review).

The solutions to the cusp/core problem can be distinguished (similarly to the solutions to other small scale problems) into cosmological and astrophysical solutions. Cosmological solutions are based on modifying the power spectrum at small scales (e.g. Zentner \& Bullock 2003), or modifying the constituent particles of DM (Colin, Avila-Reese \& Valenzuela 2000; Sommer-Larsen \& Dolgov 2001; Hu, Barkana \& Gruzinov 2000; Goodman 2000; Peebles 2000; Kaplinghat, Knox, \& Turner, M. S. 2000). Otherwise, modified gravity theories, like $f(R)$ (Buchdal 1970; Starobinsky 1980), $f(T)$ (see Ferraro 2012), and MOND (Milgrom 1983a,b), can solve the problem.

The astrophysical solutions are based on the idea that some "heating" mechanism produces an expansion of the DM component of the galaxy, reducing the inner density. In this group, the main categories are: "supernova-driven flattening" (Navarro et al. 1996; Gelato \& Sommer-Larsen 1999; Read \& Gilmore 2005; Mashchenko et al. 2006, 2008; Governato et al. 2010; Pontzen \& Governato 2011), and dynamical friction from baryonic clumps (El-Zant et al. 2001, 2004; Romano-Diaz et al. 2008, 2009; Del Popolo 2009; Cole et al. 2011).

Concerning the second problem of the $\Lambda$ CDM model, the MSP, Klypin et al. (1999), and Moore et al. (1999) noticed that numerical simulations predicted much more subhaloes in galactic and cluster haloes. The number of satellites having circular velocities larger than Ursa-Minor and Draco were $\simeq 500$, while as it is well known the MW dSphs are much less (9 bright dSphs (Boylan-Kolchin, Bullock, and Kaplinghat 2012), Sagittarius, the LMC and the SMC). Subsequent cosmological simulations confirmed the problem (Aquarius, VL2, and GHALO simulations (Springel et al. 2008; Stadel et al. 2009; Diemand et al. 2007b)). The discovery of the ultra-faint MW satellites (Willman et al. 2005; Belokurov 2006; Zucker 2006; Sakamoto \& Hasegawa 2006; Irwin et al. 2007) mitigated the problem but it did not completely solve it.

The MSP recently has shown another feature, while analyzing the Aquarius and the Via Lactea simulations. Simulated haloes have $\simeq 10$ (Boylan-Kolchin, Bullock, and Kaplinghat 2011, 2012) subhaloes too massive and dense to host MW brightest satellites. While $\Lambda$ CDM simulations predict at least 10 subhaloes having $V_{\max }>25 \mathrm{~km} / \mathrm{s}$, the dSphs of the MW all have $12<V_{\max }<25 \mathrm{~km} / \mathrm{s}$, implying a discrepancy among simulations and the dynamics of the MW brightest dSphs (Boylan-Kolchin, Bullock, and Kaplinghat 2011, 2012). This feature of the MSP problem has been dubbed the Too-Big-To-fail (TBTF) problem ${ }^{3}$.

Also in this case, two main classes of solutions have been proposed: cosmological and astrophysical. Similarly to the case of the cusp/core problem, cosmological solutions are based on modification of the spectrum of perturbations or of the particles constituting dark matter. Astrophysical solutions are connected to baryon physics, and consider: a) the change of shape of satellites from cuspy to cored (Zolotov et al. 2012 (Z12); Brooks et al. 2013 (B13)), which makes the satellites more subject to tidal stripping effects and even subject to being destroyed (Strigari et al. 2007; Peñarrubia et al. 2010). In this picture, the present-day dwarf galaxies could have been more massive in the past, and they were transformed and reduced to their present "status" by strong tidal stripping (e.g., Kravtsov, Gnedin \& Klypin

\footnotetext{
${ }^{3}$ Too big to fail means that the simulation satellites are too big with respect to MW satellites, and there is no way to have them remain invisible.
} 
2004). b) Suppression of star formation due to supernova feedback (SF), photoionization (Okamoto et al. 2008; B13), and reionization. In particular, reionization can prevent the acquisition of gas by DM haloes of small mass, then "quenching" star formation after $z \simeq 10$ (Bullock, Kravtsov, \& Weinberg 2000; Ricotti \& Gnedin 2005; Moore et al. 2006). This would suppress dwarfs formation or could make them invisible; c) effects of a baryonic disc (Z12; B13). Disc shocking to the satellites passing through the disk produce strong tidal effects on satellites, stronger if the satellite has a cored inner profile.

In the case of the TBTF problem other solutions have been proposed. The excess of massive subhalos in MW could disappear if satellites density profiles are modeled through Einasto's profiles, or if the MW's virial mass is $\simeq 8 \times 10^{11} M_{\odot}$ instead of $\simeq 10^{12} M_{\odot}$ (Vera-Ciro et al. 2012; Di Cintio et al. 2013).

The last problem is the "angular momentum catastrophe".

$\mathrm{SPH}$ simulations of galaxies show that the angular momentum of baryons is not conserved in the collapse, with the result that baryons have just $10 \%$ of the angular momentum typical of real galaxies and that disks are too small in comparison with real disks (Navarro \& Benz 1991; Navarro \& Steinmetz 1997; Sommer-Larsen, Gelato, Vedel, 1999; Navarro \& Steinmetz 2000). This problem is usually indicated with the name of "angular momentum catastrophe". Moreover, also the specific angular momentum distribution obtained in Nbody simulations disagrees with observations (mismatch of the specific angular momentum profile).

The angular momentum catastrophe has been often connected to the over-cooling problem in CDM models (e.g., White \& Reese, 1978; White \& Frenk 1991). In absence of feedback effects heating the gas (e.g., UV background reionization, ram pressure, tidal heating), the gas contracts forming small haloes and then collapses towards the center of the system loosing all angular momentum which is transferred by dynamical friction to the DM halo (Navarro \& Steinmetz 2000). Taking into account some form of feedback, like energy feedback from supernova, reduces the problem (e.g., Sommer-Larsen, Gelato, Vedel, 1999). However, even if feedback can avoid the loss of angular momentum other problems remain, like the mismatch of the angular momentum profiles (e.g., VBS), or the fact that the scatter of the logarithm of the spin parameter of real galaxies is smaller than that in simulations (de Jong \& Lacey 2000).

Maller \& Dekel (2002) proposed a model in which the DM distribution is different from that of gas, due to gas processes. In this model, SF removes gas from small incoming haloes (giving rise to the low angular momentum component of the system) eliminating baryons with low specific angular momentum (see also Sommer-Larsen et al. 2003; Abadi et al. 2004).

However, feedback models even in the absence of substructures, and then DF, have problems in forming bulgeless galaxies (van den Bosch et al, 2002), unless there exists "selective outflows" of low angular momentum gas (D'Onghia \& Burkert 2004). Recently, Governato et al. (2010) showed that outflows from supernovae explosions, removing gas with low angular momentum produces bulge-less galaxies, with a baryonic angular momentum distribution close to that of the galaxies stellar disc, and also flat galaxy density profiles.

The previous discussion shows that the small scale problems can be solved appropriately taking into account the baryon physics. A trial to find a unifying baryon solutions to the small scale problems is that of Z12, and B13. The quoted papers use the idea of Governato et al. (2010). Z12 showed that the same model can solve the TBTF. Z12 found a correction to the velocity in the central $\mathrm{kpc}$ of galaxies to mimic the flattening of the cusp due to SF, and the enhancement of tidal stripping produced by a baryonic disc. This correction together 
with destruction effects produced by the tidal field of the baryonic disc, and the identification of subhaloes that remain dark because of inefficiency in forming stars due to UV heating, were applied by B13 to the VL2 subhaloes of the VL2 simulation (Diemand et al. 2008). As a result the number of massive subhaloes in the VL2 were in agreement with the number of satellites of MW and M31.

In the present paper, we study if the cusp/core problem, the angular momentum catastrophe, the MSP, and the TBTF problem can be simultaneously solved using baryon physics, through the model of Del Popolo (2009).

The paper is organized as follows. In Sect. 2, we summarize the model. Sect. 3 describes the results and discussion. Sect. 4 is devoted to conclusions.

\section{Model}

The model used in the following was already described in Del Popolo (2009), and Del Popolo (2012a, b) (DP12a, DP12b). Here, we give a summary.

The model discussed in DP09, DP12a, b, is an improvement to the spherical infall models (SIM) already discussed by several authors (Gunn \& Gott 1972; Bertschinger 1985; Hoffman \& Shaham 1985; Ryden \& Gunn 1987; Ascasibar, Yepes \& Göttleber 2004; Williams, Babul \& Dalcanton 2004) $)^{4}$.

However, while previous authors studied how the basic SIM of Gunn \& Gott (1972) is changed by introducing one effect at a time, as a) just random angular momentum (e.g., Williams, Babul \& Dalcanton 2004), b) just adiabatic contraction (e.g., Blumenthal et al. 1986; Gnedin et al. 2004; Klypin, Zhao, and Somerville 2002; Gustafsson et al. 2006), or c) just the effect of dynamical friction of DM and stellar clumps on the halo (El-Zant et al. 2001, 2004; Romano-Diaz et al. 2008), in our model the previous effects (adiabatic contraction, dynamical friction, random angular momentum), and others (ordered angular momentum, gas cooling, star formation (see the following)) are all simultaneously taken into account.

As already reported, Gunn \& Gott (1972) studied the self-similar collapse in an expanding universe, until "shell crossing" 5 . The evolution after "shell crossing" was studied by Gunn (1977).

In the SIM, a spherical perturbation is divided into spherical shells and its evolution followed in time. Since the initial density is larger than the critical one, each particle's trajectory oscillate through the center of symmetry. If $x_{i}$ is the initial comoving radius, each shell, and the particles in it, will expand to a maximum radius, $x\left(t, M_{\mathrm{i}}\right)$, dubbed apapsis, different for each shell. The first apapsis passage is named turn-around, radius, $x_{t a}$, or $x_{m}$. After turn-around and collapse, the trajectory oscillates through the symmetry center, shell crossing starts and the mass interior to a shell is no longer constant, together with the energy. In order to handle the effect of shell-crossing and make the problem analytically tractable an adiabatic invariant is introduced (Gunn 1977). Gunn (1977) arrived to the conclusion of such an adiabatic invariant observing that the ratio of apapsis distance to the current turn-around distance decreases with time, and so does the ratio of the period of oscillation to the time scale for halo evolution (Fillmore \& Goldreich 1984). In other words, the central shells make many oscillations before there are significant changes of the potential (Gunn

\footnotetext{
${ }^{4}$ Changes to the spherical collapse introduced by dark energy where studied in Del Popolo, Pace, \& Lima (2013a, b); Del Popolo et al. (2013).

${ }^{5}$ After a shell reaches the turn-around radius it starts to collapse encountering the inner shells which are expanding or recollapsing giving rise to crossing of distinct shells.
} 
1977; Fillmore \& Goldreich 1984), and the action variables ${ }^{6}$ associated with the particle's motion, in a potential which slowly varies, are invariant. Concerning the "goodness" of the assumption indirect proofs are the good agreement between the results of high-resolution numerical simulations and those of the SIM (e.g., Ascasibar et al. 2007).

One initially calculates the density profile at turn-around, $\rho_{t a}\left(x_{m}\right)$, (Peebles 1980; White \& Zaritsky 1992; Hoffman \& Shaham 1985), assuming mass conservation and no "shellcrossing". Then after shells start to cross, the final density profile is obtained assuming that the central potential varies adiabatically (Gunn 1977, Fillmore \& Goldreich 1984), leading to

$$
\rho(x)=\frac{\rho_{t a}\left(x_{m}\right)}{f\left(x_{i}\right)^{3}}\left[1+\frac{d \ln f\left(x_{i}\right)}{d \ln g\left(x_{i}\right)}\right]^{-1},
$$

where $f\left(x_{i}\right)=x / x_{m}$ is the collapse factor, and the turn-around radius of a shell, $x_{m}$, is a monotonic increasing function of $x_{i}$, given by

$$
x_{m}=g\left(x_{i}\right)=x_{i} \frac{1+\bar{\delta}_{i}}{\bar{\delta}_{i}-\left(\Omega_{i}^{-1}-1\right)},
$$

being $\bar{\delta}_{i}$ the density excess inside the shell (see Appendix A of DP09).

In our model, we consider systems with DM and baryons. The way in which they were introduced, and how we fixed their distribution in our study was discussed in Appendix E of DP09.

In the case of the dwarfs of VBS, the baryonic fraction was calculated by means of their data. In the case of the other objects for which we do not have direct measures the baryon fraction was fixed as in McGaugh et al. (2010). We want to stress that we did not posit two different class of objects, and the baryonic fraction obtained by VBS (see their table 2), are in agreement with those obtained by McGaugh et al. (2010) (see their table 2).

The detected baryonic fraction, $f_{d}$, is given by

$$
f_{d}=\left(M_{b} / M_{500}\right) / f_{b}=F_{b} / f_{b}
$$

where $F_{b}=M_{b} / M_{500}$ is the baryonic fraction, and $f_{b}=0.17 \pm 0.01$ (Komatsu et al. 2009) is the universal baryon fraction.

The virial masses, $M_{v i r}$, are converted to $M_{500}$ using the method used in White (2001), Hu \& Kravtsov (2003), and Lukic et al. (2009). In the case of the VBS dwarfs, the baryonic fraction was calculated by summing the disc mass, $M_{\text {disc }}$ and the gas mass, $M_{\text {gas }}$, by them calculated and by the previous mass for the virial mass, $M_{v i r}$, estimated from their data (and converted to $\left.M_{500}\right)^{7}$.

During the evolution of the perturbation and its collapse, a random angular momentum $j(r, \nu)_{\text {rand }}$, is generated by random velocities (Ryden \& Gunn 1987). Usually, in the SIM

\footnotetext{
${ }^{6}$ The radial action variables is given by $J_{\mathrm{r}}=2 \pi \int_{r_{\mathrm{a}}}^{r_{\mathrm{b}}}(d r / d t) d r$, and the angular action is the angular momentum.

${ }^{7}$ Usually structures are labeled by the their density contrast with respect to the critical density, $\rho_{c}$. The mass in a given radius encompassing the overdensity $\Delta$ is given by

$$
M_{\Delta}=\frac{4 \pi}{3} \Delta \rho_{c} R_{\Delta}^{3},
$$

$M_{500}$ is the mass enclosed in $R_{500}$, defining the radius within which the mean structure overdensity is 500 times the critical density $\rho_{c}$.
} 
papers in which angular momentum is taken into account, only the random angular momentum is considered and assigned at turn-around (Nusser 2001, Hiotelis 2002; Le Delliou \& Henriksen, 2003; Ascasibar, Yepes \& Göttleber 2004), as

$$
j_{\text {rand }}=j_{*} \propto \sqrt{G M r_{m}}
$$

or obtained by the random inner motions to the proto-structure (Ryden \& Gunn 1987; AvilaReese et al. 1998; Williams et al. 2004; Del Popolo \& Kroupa 2009). Instead of directly assigning $j_{\text {rand }}$, one can express it in terms of the ratio of pericentric, $r_{m i n}$, to apocentric radii, $r_{\max }, e=\left(\frac{r_{\min }}{r_{\max }}\right)$ (Avila-Reese et al. 1998), which is constant, and approximately 0.2 in N-body simulations ${ }^{8}$. A more detailed analysis shows that particle orbits tend to become more radial when they move to the turn-around radius, and moreover eccentricity depends from the dynamical state of the system, so that

$$
e\left(r_{\max }\right) \simeq 0.8\left(r_{\max } / r_{t a}\right)^{0.1}
$$

for $r_{\max }<0.1 r_{t a}$, (Ascasibar, Yepes \& Göttleber 2004). Random angular momentum is modeled through the Avila-Reese et al. (1998) method with the Ascasibar, Yepes \& Göttleber (2004) correction.

The other form of angular momentum is the "ordered angular momentum", $h(r, \nu)^{9}$, produced by the tidal torque of the large-scale structure on the proto-structure (Hoyle 1953; Peebles 1969; White 1984; Ryden 1988; Eisenstein \& Loeb 1995; Catelan \& Theuns 1996). The total specific angular momentum, $h(r, \nu)$, is obtained by integrating the tidal torques, $\tau(r)$, over time (e.g., Ryden 1988, Eq. 35). It is common to express the total angular momentum in terms of the spin parameter, previously described. If the system is constituted of DM and baryons, the DM, and baryonic spin parameter is given by

$$
\lambda_{\text {gas }(D M)}=\frac{L_{\text {gas }(D M)}}{M_{\text {gas }(D M)}\left[2 G\left(M_{\text {gas }}+M_{D M}\right) r_{\text {vir }}^{1 / 2}\right]},
$$

$M_{\text {gas }(D M)}$ being the gas(DM) mass inside, $r_{v i r}$, the virial radius, and $L_{g a s(D M)}$ is the angular momentum of gas(DM). The $\lambda$ parameter distribution is well described by a lognormal distribution (e.g. Vivitska et al. 2002). The way dynamical friction is taken into account is described in Appendix D of DP09, and its effects on structure formation is calculated, as in DP09, by introducing the dynamical friction force in the equation of motion (Eq. A14 of DP09).

Since dynamical friction is an important component of the model, we summarize here the model. To start with, in hierarchical models of structure formation the cosmic environment is a collisionless medium with a hierarchy of density fluctuations. Matter is organized in lumps which are part of larger groups, in a hierarchy. The gravitational field of such a system is composed of an average field, originating from the "smoothed out" distribution of mass, and a stochastic field generated from the local inhomogeneities. This last component of the field can be described through a probability density $W\left(F_{\text {stoch }}\right)$ (Chandrasekhar \& von Newman 1942; Kandrup 1989; Antonuccio-Delogu \& Atrio-Barandela 1992), where $F_{\text {stoch }}$ is the stochastic

\footnotetext{
${ }^{8}$ A value $e \simeq 0.2$ produces density profiles close to the NFW model. In Avila-Reese et al. (1998, 1999) it was fixed at 0.3 .

${ }^{9}$ The peak height $\nu$ is defined as $\nu=\delta(0) / \sigma$, where $\delta(0)$ is the central overdensity value, and $\sigma$ is the density field mass variance (Eq. B12 in DP09).
} 
force. A body of mass $M$ (e.g., galaxy) moving through a cloud of smaller lighter bodies, of mass $m$ (substructure), distributed homogeneously and isotropically is slowed down due to the so called "dynamical friction" force. A formula often used to describe this force is the Chandrasekhar's formula (Chandrasekhar 1943), and a more general treatment is that of Kandrup(1980). In the last case the force per unit mass is given by

$$
\mathbf{F}=-\mu \mathbf{v}=-\frac{\int W(F) F^{2} T(F) d^{3} F}{2<v^{2}>} \mathbf{v}
$$

being $\mu$ the coefficient of dynamical friction, $T(F)$ the average duration of an impulse of the stochastic force, $\left\langle v^{2}\right\rangle$ the typical velocity of a field particle. In the case of a homogeneous system, and using the virial theorem, Eq. (2.8) can be re-written as

$$
F=-\mu v=-\frac{4.44\left[G M_{\mathrm{a}} n_{\mathrm{ac}}\right]^{1 / 2}}{a^{3 / 2} N} \log \left\{1.12 N^{2 / 3}\right\} v
$$

being $n_{\text {ac }}$ the field particles comoving number density, $N$ the number of field particles in the system, and $a$ the expansion parameter.

In order to calculate the dynamical friction force it is necessary to know the number and distribution of field particles (substructure). As already reported, in the $\Lambda$ CDM model structure forms in a hierarchical bottom-up way. Halos contain subhalos, and the last contains sub-subhalos (e.g., VL2 simulation). The subhalos cumulative mass function can be approximated as $M_{\mathrm{sub}}=0.0064\left(M_{\mathrm{sub}} / M_{\mathrm{halo}}\right)^{-1}$ to masses of $M_{\mathrm{sub}}=4 \times 10^{6} M_{\odot}$ (Diemand, Kuhlen \& Madau 2007a; Hiotelis \& Del Popolo 2006; Hiotelis \& Del Popolo 2013) ${ }^{10}$. It is interesting to note that Ma \& Boylan-Kolchin (2004) showed that even only DM clumps (subhalos) are able to flatten a cuspy profile.

In our model, gas forms clumps, which exchange angular momentum through dynamical friction with dark matter. The mass of the clumps, $m_{\text {clump }}$, is $m_{\text {clump }} \simeq 0.01 M_{\text {halo }}$, similarly to the clumps in Cole et al. (2011) simulations. Evidences of the existence of these clumps come from observation of galaxies at high redshift (van den Bergh et al. 1996; Elmegreen et al. 2004, 2009; Genzel et al. 2011) which found clumpy structures usually refereed as "clump clusters" and "chain galaxies". Inoue \& Saitoh (2011) showed through SPH simulations that these clumps are responsible for the cusp to core transformation in a similar fashion to what described in this paper.

A fundamental "recipe" in galaxy formation is adiabatic contraction (AC) of DM haloes, produced by the baryons condensation in the proto-structures centers. AC produces a steepening of the DM density profile (Blumenthal et al. 1986; Gnedin et al. 2004; Gustafson et al. 2006), when baryons cool dissipatively and collapse to the proto-structure center, giving rise to a final baryonic mass distribution $M_{b}(r)=M_{*}+M_{\text {gas }}$, where $M_{\text {gas }}$ is the gas mass and $M_{*}$ is that of stars. Dark matter particles initially located at radius $r_{i}$ will move to a new radius $r<r_{i}$, characterized by (Ryden 1988; Flores et al. 1993)

$$
r_{i} M_{i}\left(r_{i}\right)=r\left[M_{b}(r)+M_{d m}(r)\right],
$$

where $M_{d m}$ is the final distribution of DM halo particles, and $M_{i}\left(r_{i}\right)$ the initial total mass distribution, and $M_{b}$, as reported, the final baryonic mass distribution. If the halo particles orbits do not cross, we have

$$
M_{d m}(r)=\left(1-F_{b}\right) M_{i}\left(r_{i}\right) .
$$

\footnotetext{
${ }^{10}$ The $\Lambda$ CDM model also predicts the existence of microhalos (e.g., Anderhalden \& Diemand 2013).
} 
Once $M_{i}\left(r_{i}\right)$ and $M_{b}(r)$ are given Eqs. (2.10), (2.11) can be iteratively solved to find the distribution of halo particles. Usually it is assumed that the density profile of DM and baryons is the same (Mo et al. 1998; Keeton 2001; Treu \& Koopmans 2002; Cardone \& Sereno 2005), and it is given by a NFW profile. The final distribution of baryons is assumed to be a disk (for spiral galaxies) (Blumenthal et al. 1986; Flores et al. 1993; Mo et al. 1998; Klypin et al. 2002; Cardone \& Sereno 2005) ${ }^{11}$.

The previous model was improved following Gnedin et al. (2004), who showed that numerical simulation results are better reproduced if one assumes the conservation of the product of the mass inside the orbit-averaged radius, and the radius itself

$$
M(\bar{r}) r=\text { const. }
$$

where the orbit-averaged radius is

$$
\bar{r}=\frac{2}{T_{r}} \int_{r_{\min }}^{r_{\max }} r \frac{d r}{v_{r}}
$$

where $T_{r}$ is the radial period ${ }^{12}$.

The previous model does not contemplate exchange of angular momentum among baryons and DM. This is a good approximation in the early phase of the structure formation, when baryons have a density an order of magnitude smaller than that of DM, but later, when baryons density increase because of the collapse, the approximation is no longer valid. Baryons density increase acts as a coupling process of DM and baryons (Klypin et al. 2001; Klypin, Zhao, and Somerville 2002). Excitation of spiral waves and/or the presence of bar like modes can give rise to a non-axisymmetric component. The coupling effect is very powerful in the last period of structure formation, with a reduction of DM density by a factor of ten (Klypin, Zhao, \& Somerville 2002; DP09; DP12a, b).

Similarly to other semi-analytical models, following the philosophy of the White \& Rees (1978) and White \& Frenk (1991) works, we include other important physical processes such as gas cooling, and star formation (see the following).

The structure formation in our model can be summarized as follows. The galaxy formation starts with the proto-structure in its linear phase, containing DM and gas. In our model the baryons are initially in the form of a diffuse gas, with the previously quoted baryonic fraction. In order to follow the structure formation, we divide the proto-structure into mass shells, made of DM and baryons. The proto-structure evolution is followed in the expansion phase, until turn-around, and in the following collapse by means of the SIM. As is known, DM collapse earlier than baryons to form the potential wells in which baryons fall, and are subject to radiative processes with the formation of clumps, which collapse to the halo center condensing into stars, as described in Li et al. (2010) (Sect. 2.2.2, 2.2.3), De Lucia \& Helmi (2008), respectively. During the baryons infall phase, DM is compressed (AC). At this epoch the density profile of the proto-structure steepens. In their travel towards the center, baryons clumps are subject to DF from the less massive DM particles. This produces a predominant motion of DM particles outwards. The effect of the previous mechanism is amplified by the angular momentum acquired by the proto-structure through tidal torques (ordered angular

\footnotetext{
${ }^{11}$ In DP09, the final baryons distribution of typical spiral galaxies (e.g., MW, and M31) was determined using the Klypin et al. (2002) model (see their subsection 2.1), and in the case of elliptical galaxies and clusters it was assumed an Hernquist model (Rix et al. 1997; Keeton 2001; Treu \& Koopmans 2002).

${ }^{12}$ Gnedin et al. (2004) used $M(\bar{r}) r=$ const instead of $M(\bar{r}) \bar{r}=$ const because the first one is a better approximation to their simulations result.
} 
momentum), and by random angular momentum. The cuspy profile is flattened to a cored one.

Before going on, we would like to spend some words on the reasons the model used here is valuable (a deeper discussion on this issue can be found in DP09, and DP12a). Nowadays, structure formation is mainly studied through numerical simulations. Their strength is the ability to entrap to a large extent the complexity of non-linear processes. This is also their point of weakness because it is complicated to un-knot and interpret the dynamical processes they take into account in terms of the implicit physics. In this respect, semi-analytic models are much more flexible than simulations, making it possible to separate the effects of physical processes. Structure formation in simulations develops by the aggregation and merging of subclumps of matter, while in models like the SIM, haloes are spherically symmetric, they do not suffer major mergers, and accretion happens in a quiescent way. Nevertheless, the SIM describes properly structure formation. A paper of Zaroubi et al. (1996), showed that in energy space the collapse happens in a gentle way, very different from the chaotic collapse seen in N-body simulations. Several following papers converged on this point (Toth \& Ostriker 1992; Moore et al. 1999; Huss et al. 1999a, b). Moreover, the commonness of spiral galaxies, with their thin, and fragile discs testify against the primary role of major mergers in their formation (e.g., Toth \& Ostriker 1992). This point of view is confirmed by recent simulations that showed that at high redshift disc galaxies form through smooth accretion (Dekel et al. 2009). Moreover, several studies compared the SIMs results against high-resolution numerical simulations (e.g., Ascasibar et al. 2004, 2007), and showed that density profiles obtained by the SIM are in good agreement with N-body simulations. Furthermore, particle discreteness effects in dissipationless N-body simulations has been long debated (see Romeo et al. 2008), and even if nowadays these effects are "under control (see Diemand et al. 2004) the quoted simulations do not incorporate baryons. Also SPH simulations are not devoid from problems. A recent paper of Marinacci et al.(2013) shows that the high resolution SPH simulations of the formation of spiral galaxies have several drawbacks, starting from a not clear understanding of the details of the physics, to modeling and numerics involved in those calculations. Consequently alternative approaches (e.g., the SIM) may assume an important role.

Our model, is able to deal with the baryonic processes shaping the inner structure of clusters (and galaxies). It was able to predict in advance of SPH simulations (e.g., Governato et al. 2010, 2012; Martizzi et al. 2012) the correct shape of the density profiles of clusters (DP12a) and galaxies (DP09; Del Popolo \& Kroupa 2009; DP12b), and correlations among several quantities in clusters of galaxies (DP12a) later observed in Newman et al. (2013a,b). The model also predicts correctly that the inner slope of the density profiles depends on the halo mass (DP10, DP11), later seen in the SPH simulations of Di Cintio et al. (2013, 2014).

\section{Results and discussion}

As discussed in the Introduction, the small scale problems of the $\Lambda \mathrm{CDM}$ model can be solved using cosmological or astrophysical solutions. The astrophysical solutions based on the role of baryons in structure formation, are more easy to constrain than cosmological solutions, and moreover do not request one to reject the $\Lambda$ CDM paradigm for a new one.

In this section, we show how the model previously discussed changes the density profile, the angular momentum distribution in galaxies, and the galactic subhaloes distribution and characteristics. As done in previous papers, DP09, DP12a, DP12b, we will study the evolu- 

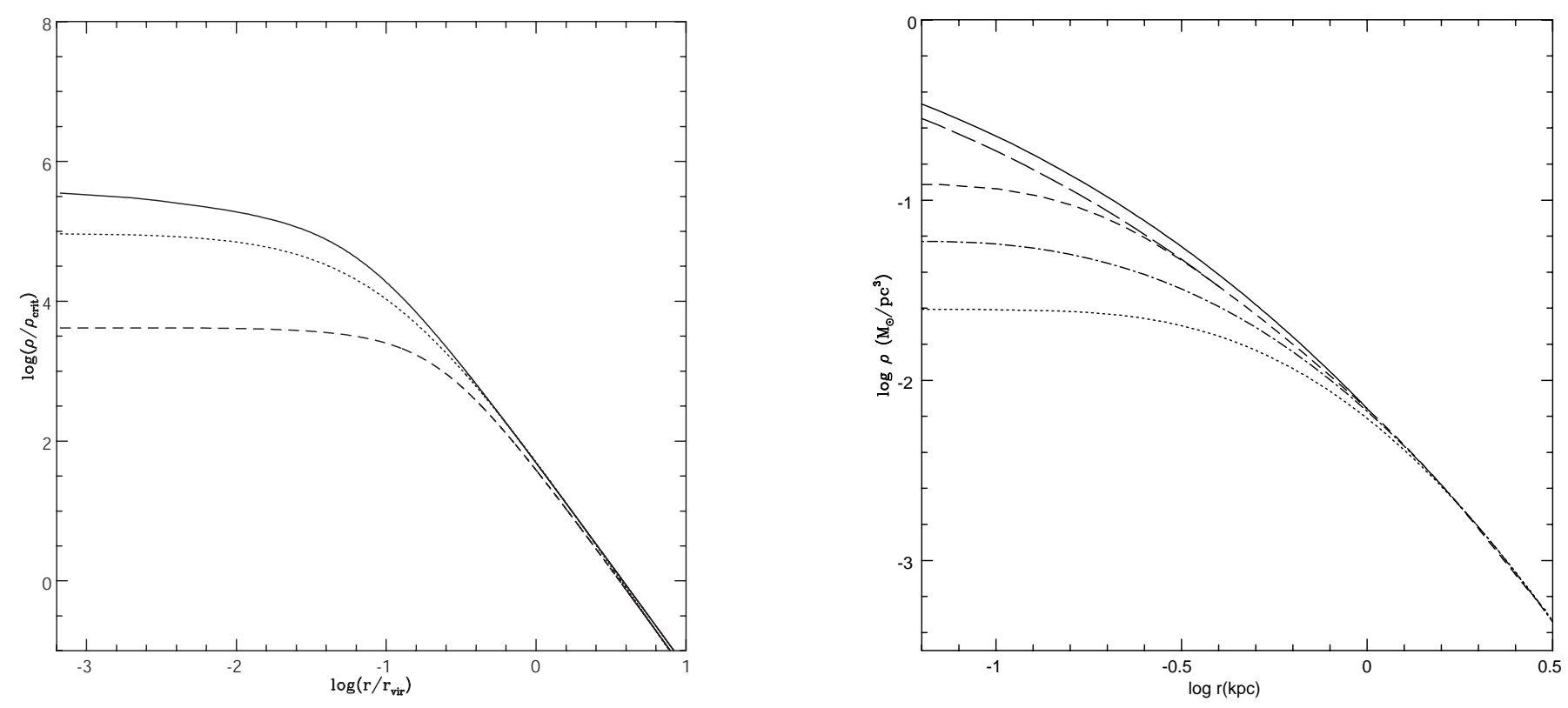

Figure 1. Fig 1a: density profiles of galaxies with mass $10^{8} M_{\odot}$ (dashed line), $10^{9} M_{\odot}$ (dotted line), and $10^{10} M_{\odot}$ (solid line). The halo with mass $10^{9} M_{\odot}$ has a baryon fraction $f_{d} \simeq 0.04$ and a specific angular momentum $h \simeq 400 \mathrm{kpc} \mathrm{km} / \mathrm{s}(\lambda \simeq 0.05)$, as in the case of UCG 6446. Fig. 1b: density profile evolution of a $10^{9} M_{\odot}$ halo starting from $z=10$ (solid line), when the profile is a NFW profile. The following evolution represented by the long-dashed line, short-dashed line, dot-dashed line, dotted line, represents the profile at $z=3,2,1,0$, respectively.

tion of protostructures from the linear phase until they form structures with galactic mass, and then we calculate their density profiles, and the DM and baryon angular momentum distribution. Then we find, similarly to Z12, an analytical correction to apply to the center of the haloes which mimics the effect of flattening of the cusp, and finally similarly to B13, we apply the previous correction together with the tidal destruction and UV heating effects on subhaloes, to the VL2, as done by B13.

\subsection{Cusp/core problem}

We start with the Cusp/core problem. In Fig. 1a, we plot the density profile of a galaxy with $M=10^{8} M_{\odot}$ (dashed line), $10^{9} M_{\odot}$ (dotted line), and $M=10^{10} M_{\odot}$. The specific angular momentum was chosen similar to one of the VBS galaxies, namely UGC 6446, to be having $h \simeq 400 \mathrm{kpc} \mathrm{km} / \mathrm{s}(\lambda \simeq 0.05)$, and the baryon fraction (see Sec. 2) is $f_{d} \simeq 0.04$. The final density profile is well described by a Burkert profile

$$
\rho(r)=\frac{\rho_{0}}{\left(1+r / r_{0}\right)\left[\left(1+\left(r / r_{0}\right)^{2}\right]\right.}
$$

being $r_{0}$ and $\rho_{0}$, the scale radius, and the central density, respectively.

A comparison of the density profiles produced by the model with the Governato et al. (2012) result, showing a good agreement with SPH simulations, was carried out in DP12a.

As Fig. 1b shows, the density profile shape is dependent on mass. The slight steepening of the density profile with mass was explained in DP09, DP10, DP12a. In summary, objects 



Figure 2. Dependence of the density profiles shape on angular momentum and dynamical friction. The symbols are like in Fig. 1. Starting from Fig. 1a, the value of the specific ordered angular momentum is changed from $h$, to $2 h$ (panel a), and from $h$, to $h / 2$ (panel b), leaving $\mu$ unchanged. The value of $\mu$ is changed to $2 \mu$ (panel c), and to $\mu / 2$ (panel d), leaving $h$ unchanged.

less massive originate from a smaller peak height $\nu$. Since, the specific ordered (h), and random $\left(j_{\text {rand }}\right)$, angular momentum acquired by the protostructure are anticorrelated with $\nu$, smaller objects acquire larger angular momentum. "Particles" of the protostructure having larger angular momentum, will remain for a longer time close to maximum radius, giving rise to shallower profiles (see also Williams et al. 2004). As described in DP12a,b, there exists a correlation between the angular momentum acquired by the structure, the baryon content of the structure, and the shape of the density profile. Then the larger is the angular momentum acquired by the galaxy, the flatter is the density profile (see also DP09; DP12a).

The steepening of the density profile with mass is in agreement with density profiles of THINGS galaxies (de Blok et al. 2008). de Blok et al. (2008) found that brighter, and larger galaxies, with $M_{B}<-19$ have density profiles well described both from cored and cuspy profiles, while cored profiles are preferred for less bright, and less massive galaxies, with $M_{B}>-19$. 
Fig. 1b, shows the evolution of a $10^{9} M_{\odot}$ halo starting from the redshift of virialization $z=10$ (solid line). The density profile at $z=3,2,1$, and 0 , is represented by the long-dashed line, short-dashed line, dot-dashed line, and dotted-line, respectively. The proto-structure and profile evolution was summarized in the final part of Sect. 2. After virialization the density profile evolution is due to secondary infall, two-body relaxation, interplay between $\mathrm{DM}$ and baryons. As shown in Fig. 1b, the profile flattens and a core is formed within 1 kpc. A similar result was obtained by Romano-Diaz et al. (2008), who performed N-body simulations of the DM density profile in the absence and in the presence of baryons. Cusp erasing was connected by them to an influx of sub-haloes in the central part of the halo, as well as to heating up of DM through DF, similarly to the El-Zant et al. (2001, 2004) results. Concerning the model proposed by El-Zant, it is based on baryonic clumps which exchange angular momentum through dynamical friction with DM. As previously reported, there are observational evidences on the existence of these clumps, coming from high- $z$ observations of galaxies (van den Bergh et al. 1996; Elmegreen et al. 2004, 2009; Genzel et al. 2011). These clumps are of fundamental importance in the formation of bulges, in the so called "clump-origin bulge" (Noguchi 1998, 1999; Inoue \& Saitoh 2012). Inoue \& Saitoh (2011), showed through SPH simulations that these clumps are able to flatten the cuspy profile.

Theoretical results in agreement with the previous ones are those of Ma \& BoylanKolchin (2004), Nipoti et al. (2004); Romano-Diaz et al. (2008, 2009)); Cole et al. (2011). Cole et al. (2011) showed that the mechanisms described by the previous quoted authors are very efficient in cusp erasing.

We hinted to the role of angular momentum on the density profiles shapes. Another quantity on which the model depends is the "magnitude" of dynamical friction. The dynamical friction force can be written (see Eq, D5 in DP09) as

$$
F=-\mu v
$$

where $\mu$ is the coefficient of dynamical friction. The effect of changing this quantity is similar to changing the magnitude of the angular momentum. Namely, increasing $\mu$ gives rise to shallower profiles.

In Fig. 2, we plot how the density profiles change when $h$, and $\mu$ are changed. Fig. 2a plots the density profiles in Fig. 1a when $h$ is increased to $2 h$, leaving $\mu$ unchanged. In Fig. $2 \mathrm{~b} h$ is decreased to $h / 2$. As already reported, the increase in the magnitude of the angular momentum flattens the profile, and its decrease produces a steepening of the profile. In Fig. $2 \mathrm{c}$, the value of $\mu$ is increased to $2 \mu$, and in Fig. $2 \mathrm{~d}$ it is reduced to $\mu / 2$, leaving unchanged the angular momentum. As already reported, the effect of dynamical friction is similar to that of angular momentum, but its effect is slightly lower.

\subsection{Angular momentum catastrophe}

In the following, we study the angular momentum distribution (AMD) of the structure obtained with the method described in Sect. 2 and compare it with that obtained by VBS using 14 dwarfs. Before, we recall some theoretical background.

A virialized halo made of baryonic matter and DM of virial mass $M_{v i r}$ has a total specific angular momentum $(\mathrm{SAM}), j_{\text {tot }}=L_{t o t} / M^{13}, L$ being the angular momentum, proportional

\footnotetext{
${ }^{13}$ Note that the SAM $\mathrm{j}$ used here is the total SAM, sum of h, and $j_{\text {rand }}$ seen in Sect. 2 .
} 


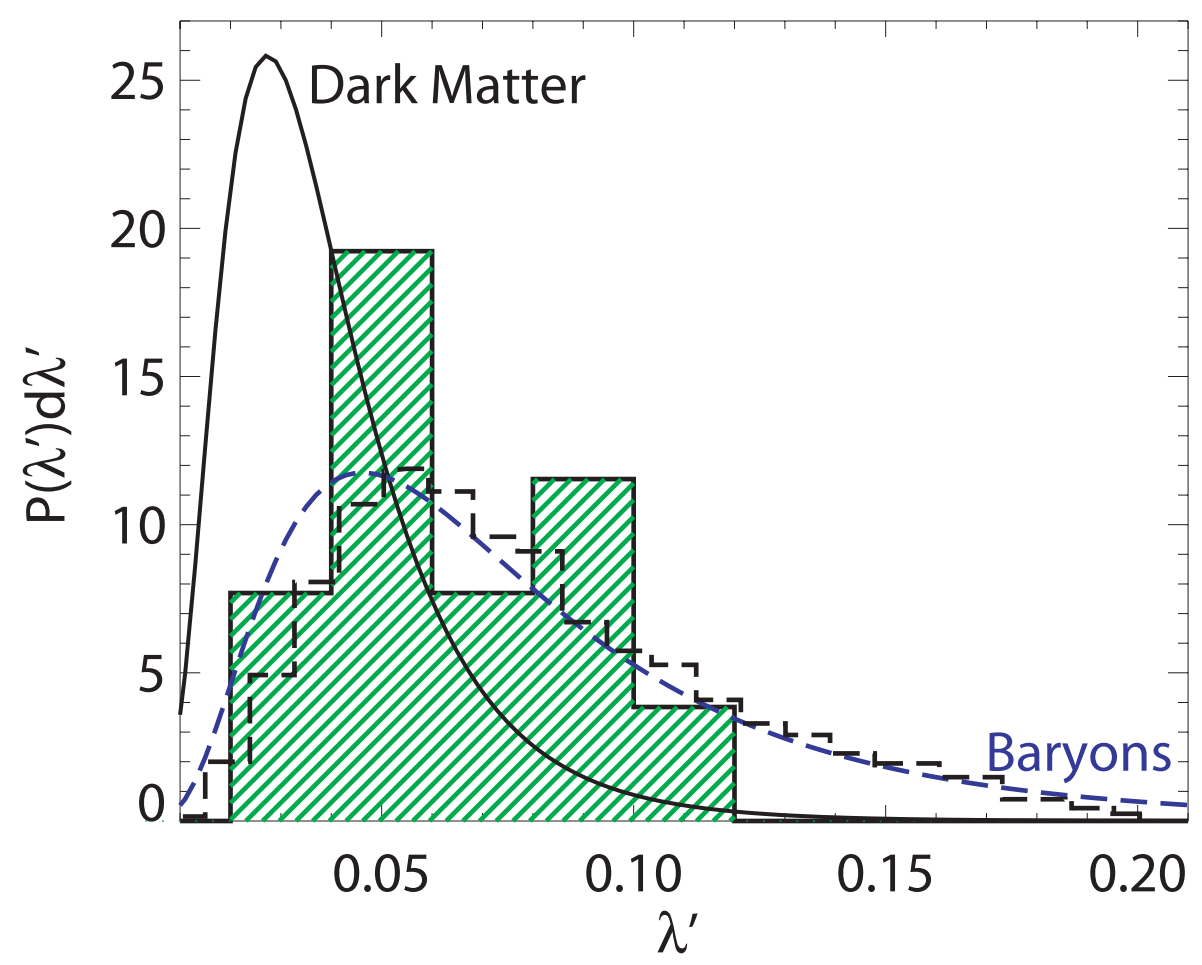

Figure 3. The distribution of $\lambda^{\prime}$ in the sample of dwarf galaxies by VBS (shaded histogram) compared to the Maller \& Dekel (2002) predictions for the baryons in dwarf galaxies (dashed curve), and to N-body simulation result for dark halos by Bullock et al. (2000) (solid curve). The dashed histogram represents our result.

to the maximum SAM, $j_{\max }$, and the normalized cumulative distribution, $m(j)$

$$
j_{\text {tot }}=j_{\max }\left[1-\int_{0}^{1} m(k) \mathrm{d} k\right] \text {, }
$$

where $k=j / j_{\max }$, and

$$
m(j)=\int_{0}^{j} p(j) \mathrm{d} j .
$$

$p(j) d j$ being the mass fraction having SAM in the $j-j+d j$ range. A quantity often used to express the angular momentum, $L$ of a structure, is the spin parameter

$$
\lambda=L \sqrt{|E|} / G M^{5 / 2}
$$

(Peebles 1969), where E indicates the system internal energy. For practicality reasons, the previous spin parameter definition is substituted by

$$
\lambda=\frac{j_{\text {tot }}}{\sqrt{2} r_{\text {vir }} V_{\text {vir }}},
$$

where $V_{\text {vir }}=\sqrt{G M_{\text {vir }} / r_{\text {vir }}}$, and $r_{\text {vir }}$ is the virial radius. For a NFW profile, $\lambda^{\prime}=\lambda / \sqrt{f_{c}}$, and $f_{c}$ is a function of the concentration parameter $c_{v i r}$ (Mo et al. 1998). As shown by Bullock et al. (2000), the AMD is well described by

$$
m(k)=\frac{\mu k}{k+\mu-1} .
$$


Bullock et al. (2000) showed that for a $\Lambda$ CDM Universe with $\sigma_{8}=1.0, h=0.7, \Omega_{m}=$ $0.3, \Omega_{\Lambda}=0.7, \mu$ has a Gaussian distribution in $\log (\mu-1)^{14}$. The probability distribution corresponding to Eq. (3.7) is given by

$$
p(t)=\frac{\xi \mu(\mu-1)}{(\xi t+\mu-1)^{2}},
$$

where $t=j / j_{\text {tot }}$, and $\xi$ is the ratio of the total and maximum SAM, $\frac{j_{\text {tot }}}{j_{\max }}$

$$
\xi=\frac{j_{\text {tot }}}{j_{\max }}=1-\mu\left[1-(\mu-1) \ln \left(\frac{\mu}{\mu-1}\right)\right] .
$$

Going back to VBS, in their study they fitted the rotation curves of 14 dwarfs (previously studied by VBS), assuming that the galaxies are constituted by a NFW dark halo, a thin gas disc, and a thick stellar disc. They obtained the concentration parameter, $c_{v i r}$, the virial velocity, $V_{v i r}, f_{d i s c}=M_{d i s c} / M_{v i r}$, and $f_{\text {gas }}=M_{\text {gas }} / M_{\text {vir }}$. The SAM distribution was obtained through $m(j)=M_{\text {disc }}(r) / M_{\text {disc }}\left(r_{\max }\right)$, where $j=r V_{c}(r)$. By means of Eq. (3.3) they got $j_{\text {tot }}$ for each galaxy and from this the disc spin parameter

$$
\lambda_{\text {disc }}=\frac{j_{\mathrm{tot}}}{\sqrt{2} r_{\mathrm{vir}} V_{\mathrm{vir}}},
$$

Finally, they compared the histograms of $\lambda_{\text {disc }}$ distribution for the quoted galaxies with the $\lambda$ distribution of DM haloes (that they found agreement, in contrast with Maller \& Dekel 2002 , Governato et al. 2010), and the $j$ distribution, $p(t)$, that was compared to the DM distribution. In their analysis, they assumed that the mass-to-light ratio was 1 in the $\mathrm{R}$ band ${ }^{15}$.

We repeated their analysis as follows. We used the gas fraction, total SAM, obtained by them, and estimated the mass of each galaxy from their $V_{v i r}$, assuming like them that the density profile was a NFW one ${ }^{16}$. Then, by means of the model in Sect. 2, similarly to DP12a, we "simulated" each galaxy. Then, similarly to VBS we obtained the spin parameter distribution, and the $j$ distribution. Improved results could be obtained using the VBS rotation curve data, and use a Burkert profile for the DM halo.

Like them, we calculated the cumulative AMD, $m(j)=M_{d i s c}(r) / M_{d i s c}\left(r_{\max }\right)$, we got through Eq. (3.3) $j_{\text {tot }}$, and then the spin parameter. Finally, we obtained the $p(t)$ of the AMDs.

The results are reported in Fig. 3, and Fig. 4. In Fig. 3, we plotted the $\lambda^{\prime}$ distribution. The solid line represents the results of Bullock et al. (2000) for the DM spin parameter distribution, while the dashed curve, the spin parameter distribution for the baryons in the 14 VBS galaxies obtained by Maller \& Dekel (2002). The solid histogram is the spin distribution obtained from the 14 VBS galaxies (see Maller \& Dekel 2002), and the dashed histogram is the spin parameter distribution obtained from the 14 VBS galaxies, using the method of this paper.

The plot shows that the spin distribution of DM (solid line) is different from that of galaxies (solid histogram). The spin distribution obtained by Maller \& Dekel (2002) (dotted

\footnotetext{
${ }^{14}$ The mean is -0.6 and the standard deviation 0.4 . For $90 \%$ of the haloes the value of $\mu$ is $1.06<\mu<2.0$ with $\langle\mu\rangle=1.25$.

${ }^{15}$ In any case, the results are not sensitive to this choice, since dwarfs are DM dominated.

${ }^{16}$ For a NFW profile, the virial mass is given by $M_{v}=\sqrt{\frac{3}{800 \pi \rho_{c} G^{3}}} V_{v}^{3}=\sqrt{1 / 100}\left(H_{0} G\right)^{-1} V_{v}^{3}$.
} 

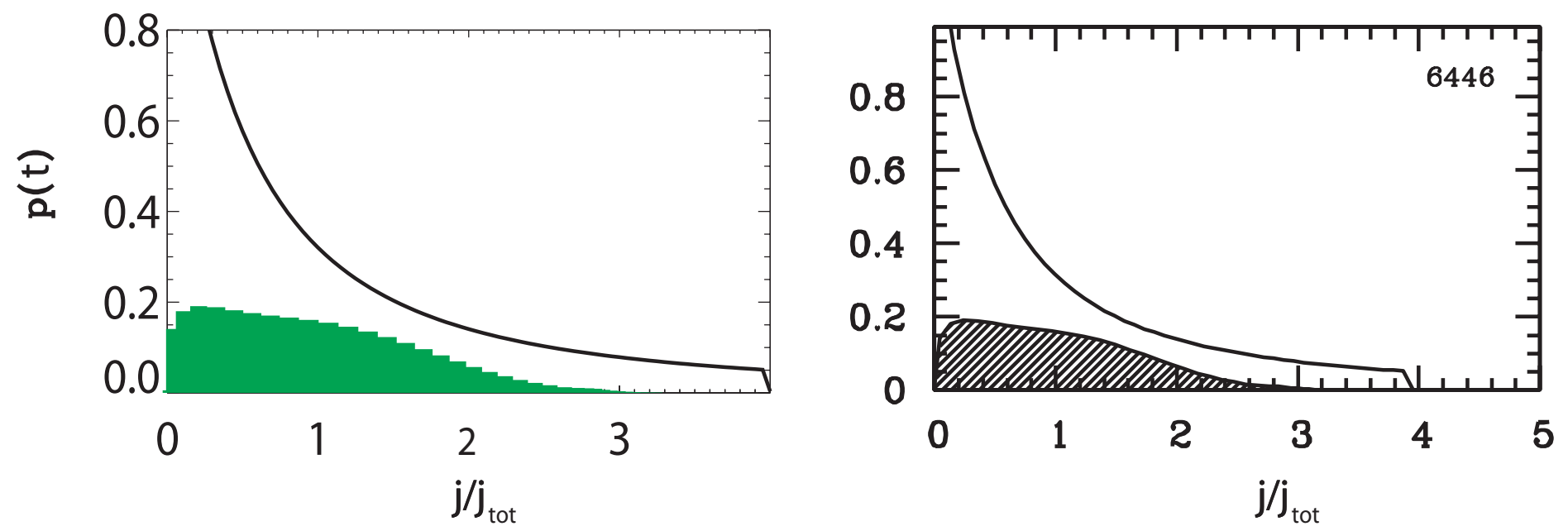

Figure 4. The $p(t)$ of the AMDs for one of the 14 galaxies of VBS (shaded area) compared to the $p(t)$ of Eq. (3.8) with $\mu=1.25$ (normalized to unity) (solid line), namely the median of the LCDM haloes AMDs. In the left panel, we plot the result of our calculation, while in the right panel we plot VBS result for UGC 6446. The difference between the two distributions is connected to the AMD of baryons not incorporated in the disc (under the standard hypothesis of conservation of SAM of baryons). Preferentially highest and lowest momenta baryonic matter is absent in the disc.

curve), and by us (dashed histogram), are in good agreement with the observational distribution of the spin parameter in galaxies. The Maller \& Dekel (2002) feedback model produces a good agreement with observations. As reported by them, the agreement is independent of the feedback model used.

In Fig. 4, we plot the $j$ profile for UGC 6446 obtained by VBS (Fig. 4b), and the one calculated by us (Fig. 4a). In the figure, the solid line is the Bullock et al. (2001) average DM j-profile $(\mu=1.25)$, while the shaded area represent the $p(t)$ of AMD for UCG 6446. The figure shows the angular-momentum profile mismatch. The dwarf angular momentum shows a deficit of angular momentum, at the high and low end of the distribution, with respect to the DM distribution. In models like that of Maller \& Dekel (2002) or simulations like that of Governato et al. (2010), the low-j tail in the distribution is missing because feedback (blowout) selectively removes gas from small satellites, giving rise to the material of the halo having low-j.

In our model, the reduction of DM density in the inner part of haloes (previously described) and the change of the angular momentum distribution, is due to the following reasons.

Already more than a decade ago, semi-analytic models of galaxy and disc formation of isolated halos predicted the correct size of discs and of disc galaxy formation (e.g., Mo, Mao, \& White 1998; van den Bosch 2002; Natarajan 1999; Firmani \& Avila-Reese 2000; Buchalter, Jimenez, \& Kamionkowski 2001; Mayer, Governato, \& Kaufmann 2008). The models assumed that a) angular momentum is conserved during the collapse phase of baryons inside halos, that b) baryons have the same initial specific angular momentum distribution of DM. Vice versa, only recently it was understood that a necessary condition to form realistic galaxies and discs in simulations is to have high spatial and mass resolution (Mayer, Governato, \& Kaufmann 2008; Governato et al. 2010). This avoid "spurious" dissipation of angular momentum that can be produced by the interaction of particles having noteworthy tempera- 


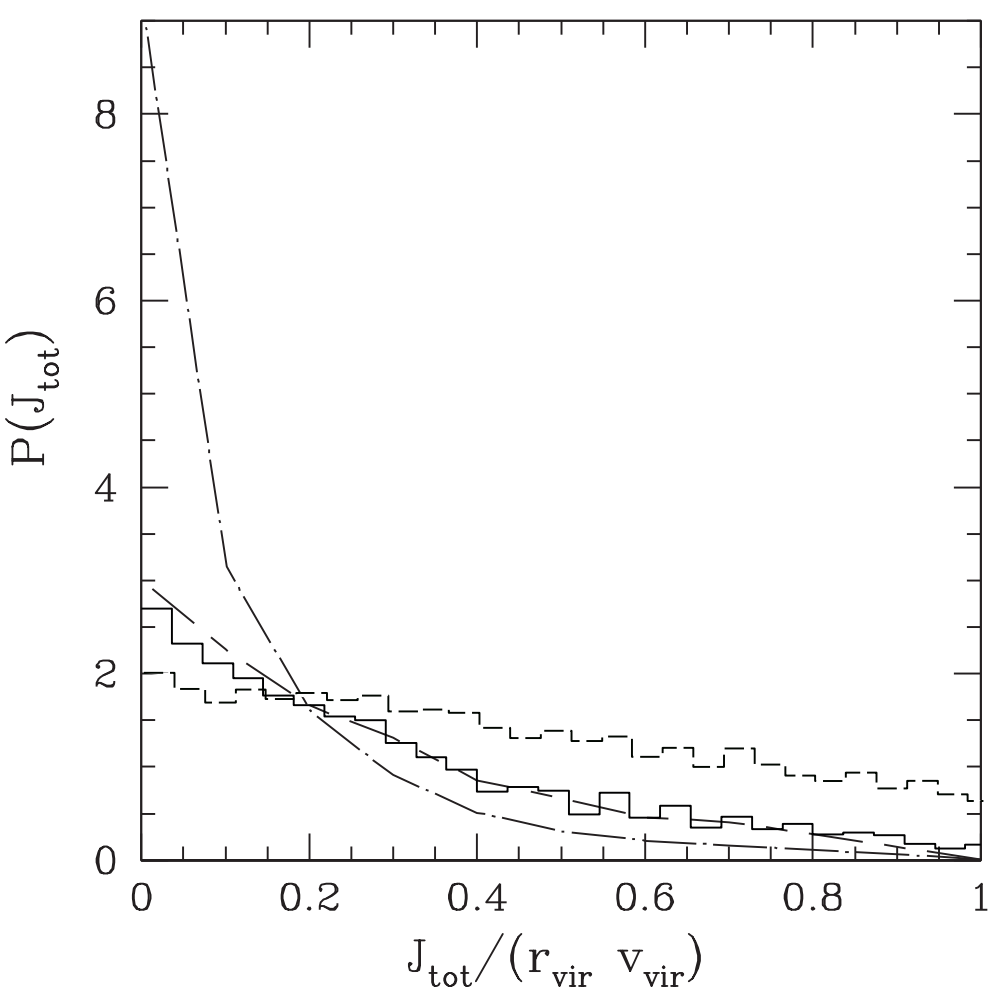

Figure 5. Total SAM distributions. The distribution of angular momentum in the halo n. 081 (dashed line), and in the halo n. 171 (dot-dashed line) of van den Bosch et al. (2002) compared with the SAM of a $10^{12} M_{\odot}$ halo obtained with our model (dashed histogram), and that for the density profile reproducing the NFW halo (solid histogram).

ture difference or by artificial viscosity. Moreover, during merging angular momentum is lost through DF. As before reported, the previous problem can be solved if the thermal energy of baryons is enough to resist collapse, changing the merging hierarchy dictated by dark matter, and forming a smoother distribution. If gas is not clumpy, but has a smooth distribution, this reduces the angular momentum loss caused by DF.

In our semi-analytic model, structure formation undergoes quescient accretion, and minor mergers, differently from simulations in which structures are obtained as a result of major mergers of sub-haloes. The existence of many spiral galaxies with thin disks somehow supports the previous quiescent formation mechanism. Moreover, Dekel et al. (2009), argued that disc galaxies, at high $z$, are not formed through galactic mergers, but by smooth accretion flows. Consequently, the angular momentum loss to which simulations are exposed, is not present.

This can be shown, as already done in DP09, and Williams et al. (2004), as follows. In Fig. 5 the dot-dashed line is the specific angular momentum distribution of the halo $\mathrm{n}$. 170 of van den Bosch et al. (2002) (resembling most of the SAMs in van den Bosch et al. 2002). The dashed line represents the specific angular momentum distribution of the halo n. 81 of van den Bosch et al. (2002) (shallowest distribution found in their simulations). The dashed histogram represents the SAM of a halo of $10^{12} M_{\odot}$ obtained with our model. Starting from the $10^{12} M_{\odot}$ halo obtained in our model, we reduced the angular momentum in order to reproduce a NFW profile having $10^{12} M_{\odot}$, and $c=10$. Then we calculated the 
SAM for this halo, which is the solid line histogram in Fig. 5. This last histogram is closer to the typical halos coming out from numerical simulations than the histogram relative to our halo. Moreover, the histogram of the SAM of the halo reproducing the NFW profile (solid histogram) is more centrally concentrated than the SAM of the halo of the initial halo (dashed histogram). Fig. 5 suggests that halos in simulations loose angular momentum between $0.1 r_{\text {vir }}$ and $1 r_{\text {vir }}$, when compared to the halos obtained in our model, or in semianalytic models (see Williams et al. 2004), in agreement with the "angular momentum catastrophe"

Similarly to what happened with the semi-analytic model previously cited (e.g., Mo, Mao, \& White 1998), halos and galaxies forming in our model are closer to real halos and galaxies than those generated in simulations, at least closer that those generated in simulations of some years ago. As previously reported, recently, increasing the simulations resolution and properly using feedback, simulations are able to form realist galaxies (e.g., Mayer, Governato, \& Kaufmann 2008; Governato etl a. 2010).

Another important issue in the solution of the "angular momentum catastrophe" in our model is the fact that angular momentum is mainly lost from the smaller baryonic clumps. As reported in Sec. 3.1, the "ordered" angular momentum, $h$, acquired by an halo is anticorrelated with the peak height $\nu$, and consequently smaller haloes acquire larger angular momentum. Similarly, the "random" angular momentum $j$ is anticorrelated with $\nu$ (see Eq. C13, and Fig. 8 in DP09). Consequently, smaller baryonic clumps inside the halo have larger angular momentum ("random" angular momentum) than larger clumps. In the virialization process, these clumps loose more angular momentum than larger clumps, explaining why the low tail in the AMD is missing in Fig. 4.

\subsection{The Too Big to Fail and the Missing Satellites problems}

The other small scale problem of the $\Lambda$ CDM model is the MSP, concerning the incorrect prediction of simulations of the subhaloes distribution in structures or satellites in our MW. The TBTF problem is a peculiar feature of the MSP, which showed that subhalos in a DMonly simulation have significantly higher central densities than inferred from observations of the Milky Way's classical dwarf spheroidal satellites.

Several solutions have been proposed to both problems (Strigari et al. 2007; Simon \& Geha 2007; Madau et al. 2008; Zolotov et al. 2012; Brooks \& Zolotov 2012; Purcell \& Zentner 2012; Vera-Ciro et al. 2012; Di Cintio et al. (2012); Wang et al. 2012). B13, proposed an interesting baryonic solution to the MSP and the TBTF problem. Namely, instead of running SPH simulations of different galaxies, they tried to introduce the baryons effect in large N-body dissipationless simulations, like the VL2, showing that the result obtained is in agreement with observations of MW and M31 satellites.

In the following, we will partly follow their steps to obtain the corrected circular velocities and distribution of VL2 satellites in the framework described in Sect. 2.

In summary the method is based on the following ideas and is divided into two main phases.

Initially the attention is directed to the isolated satellites, before accretion to the main system. As noticed by several authors (e.g., Mashchenko et al 2006, 2008; Peñarrubia et al. 2010) the effects of the tidal forces of the main halo on a satellite depends fundamentally on its shape. If the satellite has a cuspy profile, its structure will not suffer big changes, when it will enter the main halo. If the satellite has a cored profile, the tidal field of the main halo can strip easily its gas and in some cases even destroy it (Peñarrubia et al. 2010). So, this 
first phase, defining the shape of the satellite is of fundamental importance. This first phase and the flattening of the satellites density profiles will be studied through our model.

Then comes the second phase, when the satellite is no longer considered isolated, it is subject to the tidal field of the main halo, and finally it is accreted to the main halo (see the following).

Our result for the flattening of the density profile (first phase), is expressed in terms of the change to circular velocity in $1 \mathrm{kpc}$, as in Z12, and is given by

$$
\Delta\left(v_{1 k p c}\right)=0.3 \mathrm{v}_{\text {infall }}-0.2 \mathrm{~km} / \mathrm{s}
$$

in the $20 \mathrm{~km} / \mathrm{s}<\mathrm{v}_{\text {infall }}<50 \mathrm{~km} / \mathrm{s}$ interval. We want to stress again that the previous correction is determined for the Z12 satellites, and this correction will be applied to VL2, together with the other corrections discussed in the following. The correction that we obtained is close to that obtained by Z12, accounting mainly for the reduction of the subhaloes central mass produced by $\mathrm{SF}$, and given by

$$
\begin{aligned}
\Delta\left(v_{1 k p c}\right)= & 0.2 \mathrm{v}_{\text {infall }}-0.26 \mathrm{~km} / \mathrm{s} \\
& 20 \mathrm{~km} / \mathrm{s}<\mathrm{v}_{\text {infall }}<50 \mathrm{~km} / \mathrm{s}
\end{aligned}
$$

The last equation gives the difference between DM and SPH runs, and then the corrections to apply to satellites in N-body simulations that take account of the missing piece of baryonic physics.

In the case of a $v_{\text {infall }}=30 \mathrm{~km} / \mathrm{s}$, the Z12 correction gives $\Delta\left(v_{1 k p c}\right)=5.74$, while our correction gives $\Delta\left(v_{1 k p c}\right)=8.8$. The difference between the $\Delta\left(v_{1 k p c}\right)$ of our model and that of Z12 is due to the different models used to produce the pre-infall flattening of the satellites density profile. The difference among our model and that used in Z12 is the model used to calculate the pre-infall flattening. In Z12 the pre-infall flattening is due to SF, and in our case is connected to dynamical friction. As shown by Cole et al. (2011), DF on infalling clumps is a very efficient mechanism in flattening the DM profile. A clump having a mass of $1 \%$ of the halo mass can give rise to a core from a cuspy profile removing two times its mass from the inner part of the halo. In the case of the SF the mechanism should be less effective going down to lower masses (e.g., dwarfs with stellar mass $<10^{5}-10^{7} M_{\odot}$ have few stars and supernovae explosions are as a consequence less present).

As reported by B13, the baryonic corrections taken into account by the Z12 correction, and consequently ours, reduces the number of massive satellites that are expected in dissipationless N-body simulations, but we are also interested in seeing if the baryonic corrections also reduce the number of luminous satellites, and for this other corrections are needed.

Our correction (Eq. 3.11), similarly to the Z12 correction applies to satellites that survived at $z=0$, and that had the central $V_{c}$ reduced by baryonic physics. Before $z=0$ satellites could have been destroyed (by e.g. stripping or photo-heating) (see the following). In N-body simulations, like the VL2, baryons effects are not taken into account, and then satellites that in the real universe or in SPH simulations may be totally destroyed by enhanced tidal stripping (due to disks and cores) and SF, will not be destroyed in the VL2. Since our correction and Z12 correction applies to satellites that survived to $\mathrm{Z}=0$, not those that were previously destroyed, we need two other corrections (tidal stripping, and suppression of star formation) as done by B13, to calculate the survived satellites and then apply the Z12 correction.

Here starts the second phase always mentioned, considering the effects of the interaction between the main halo and the satellite. 
In order to take account of tidal stripping, and tidal heating after infall, to get accurate $V_{\text {max }}$ values, we could follow the Taylor \& Babul (2001) model (see the following), which takes into account the effect of tidal mass loss, and tidal heating in an analytical manner. However, this will be done in a future paper, and here we estimate the effect of stripping after infall, adding two corrections as done in B13.

The first of the two corrections is the suppression of star formation by photo-heating, obtained following the Okamoto et al. (2008) results. The last one is the destruction rates by stripping of satellites, obtained following Peñarrubia et al. (2010).

We will use Eq. 8 from Peñarrubia et al. (2010), for subhaloes with density slope of dark matter $\gamma$, to calculate

$$
v_{\text {max }}(z=0)=v_{\text {infall }} \frac{2^{\alpha} x^{\eta}}{(1+x)^{\alpha}},
$$

where $x \equiv \operatorname{mass}(\mathrm{z}=0) / \operatorname{mass}(\mathrm{z}=$ infall $)$, describing the change in $V_{\max }$ as a function of mass lost to tidal stripping, and $\alpha$, and $\eta$ are two fitting parameters whose values are connected to the slope $\gamma$ as shown in Fig. 6 of the Peñarrubia et al. (2010). For the DM-only runs, which have cuspy density profiles, $\gamma=-1$ gives a good fit to the change in $V_{\max }$. Since the satellites have a profile that can be cored, we have chosen a value of $\gamma$ in the range $\gamma=-0.5 \div 0$, and values of $\alpha$, and $\eta$ given by Peñarrubia et al. (2010), Fig. 6. In the case of the VL2 satellites, the values of $\alpha$ and $\eta$ are 0.4 , and 0.3 , respectively.

In order to use the quoted equation, we need to know the mass lost in tidal stripping. Those values can be read from Fig. 6 in Z12. Then, we apply the Peñarrubia et al. (2010) equation, to get the change in $V_{\max }$. Assuming that $V_{\max }$ is approximately the same as $V_{1 k p c}$, we get the needed correction after infall.

Similarly to B13, a) the population of satellites that looses more than $97 \%$ of their infall mass, and b) all subhalos losing more than $90 \%$ of their infall mass, having also apocentric passages $<20 \mathrm{kpc}$ and $v_{\text {infall }}>30 \mathrm{~km} / \mathrm{s}$, are considered destroyed. Then the subhaloes that remain dark because they are affected by UV heating, and do not form stars, are calculated using the Okamoto et al. (2008) SPH simulations and Sect. 3.2 results of B13. Luminosity is assigned to the VL2 satellites as done in B13 (Eqs. 3, 4).

Finally, we have to assign a luminosity to the satellites which survived. We use the relation between $v_{\text {infall }}$ and the stellar mass $M_{*}$ obtained by Z12

$$
\left(\frac{v_{\text {infall }}}{\mathrm{kms}^{-1}}\right)^{6}=55.56 \frac{M_{*}}{M_{\odot}}
$$

and then using a relation between $M_{*}$ and the visual magnitude $M_{\mathrm{V}}$

$$
M_{*}=L_{\mathrm{V}} \times 10^{-0.734+1.404(B-V)}
$$

where

$$
L_{\mathrm{V}}=10^{-(V-4.8) / 2.5}
$$

Munshi et al. (2013), that we approximate as in B13 with

$$
\log _{10}\left(\frac{M_{*}}{M_{\odot}}\right)=2.37-0.38 M_{\mathrm{V}}
$$

The result of the previously corrections are plotted in Fig. 6. The top panel of this figure represents the results from VL2 at $z=0$. The bottom panel represents the same satellites 
after the previous discussed corrections (heating, destruction, and velocity corrections) were applied. The red filled symbols are the object "observable" in VL2. Dark objects are marked by empty circles. Objects marked with a circle and a " $\mathrm{x}$ " through them, represent subhaloes that do not survive to the baryonic effects (e.g., baryonic disc, etc). Empty circles have a mass smaller than the minimum to retain baryon and form stars. Finally, filled black circles are satellites that lose $90 \%$ of their mass since infall, but do not satisfy the destruction criteria previously described.

Note that the Z12 correction was not applied to satellites with $v_{\max }>50 \mathrm{~km} / \mathrm{s}$ (for example to the satellites with $M_{V}<-16$, that are the 5 most massive satellites at infall, and are the five that had $v_{\max }>50 \mathrm{~km} / \mathrm{s}$ ).

The number of satellites with $v_{1 k p c}>20 \mathrm{~km} / \mathrm{s}$ is, similarly to B13, equal to 3 . Differently from B13, our central velocities are smaller (the correction to the circular velocity, $\Delta\left(v_{1 k p c}\right)$, to subtract to the circular velocity itself, $V_{c}$, is larger in our model with respect to Z12, and B13). Also in our case some satellites are "overcorrected", namely their velocities are negative.

The reason for the quoted overcorrection is similar to that described in B13. The halos whose velocity is overcorrected, is a halo population that after infall lost a great part of their mass and so, at $z=0$, the circular velocity in $1 \mathrm{kpc}, v_{1 \mathrm{kpc}}$, is very low, giving rise to the quoted overcorrection. After infall, the quoted population lost more than $99.9 \%$ of their mass, and moreover their tidal radius is $<1 \mathrm{kpc}$. The population can be considered a population of destroyed subhaloes.

As in B13, UV heating, and tidal destruction are necessary to reconcile the total number of luminous satellites with observations, while the Z12 correction is necessary to reconcile the masses of the subhaloes with observations.

In the present section, we considered the evolution of satellites before infall, and calculated the flattening of the density profile using our model (not the SF flattening effect), and added to this the further flattening (or reduction of the central rotation velocity) produced by the interaction with the main halo. This last step was based on the calculation of the flattening, after infall, shown in B13. In a forthcoming paper, we will improve the model as follows. We calculate again the profile flattening of satellites before infall as done in this paper, but then we will use a model, like that of Taylor \& Babul (2001) able to follow the merger history and growth of the satellites while interacting with the main halo, tracking the substructure evolution in the main halo (Del Popolo \& Gambera 1997), taking account of the mass loss due to tides, and tidal heating. In this way, we have enough information to conclude if they are massive enough to retain baryons and form stars.

Since our model is not so computationally "heavy" as SPH simulations, we could study the MSP problem in different galaxies, since it is not enough to solve the problem in a single galaxy to conclude that the problem is solved in galaxies different from ours. In fact, several authors have discussed the MSP problem in relation to the host galaxy mass. Di Cintio et al. (2012), Vera-Ciro et al. (2012), Wang et al. (2012), showed that if the MW true virial mass is smaller than $10^{12} M_{\odot}$, namely $\simeq 8 \times 10^{11} M_{\odot}$, the satellites excess may disappear.

In any case, the main point of our study, even if some points of the previous results need to be improved, is that baryonic physics is able to solve simultaneously the small scale problems of the $\Lambda \mathrm{CDM}$ model: cusp/core problem, angular momentum catastrophe, MSP, and TBTF problem.

A similar result, has been obtained by a series of papers (Governato et al. 2010; Governato et al. 2012; Zolotov et al. 2012; Brooks \& Zolotov 2012). In those papers, the main 


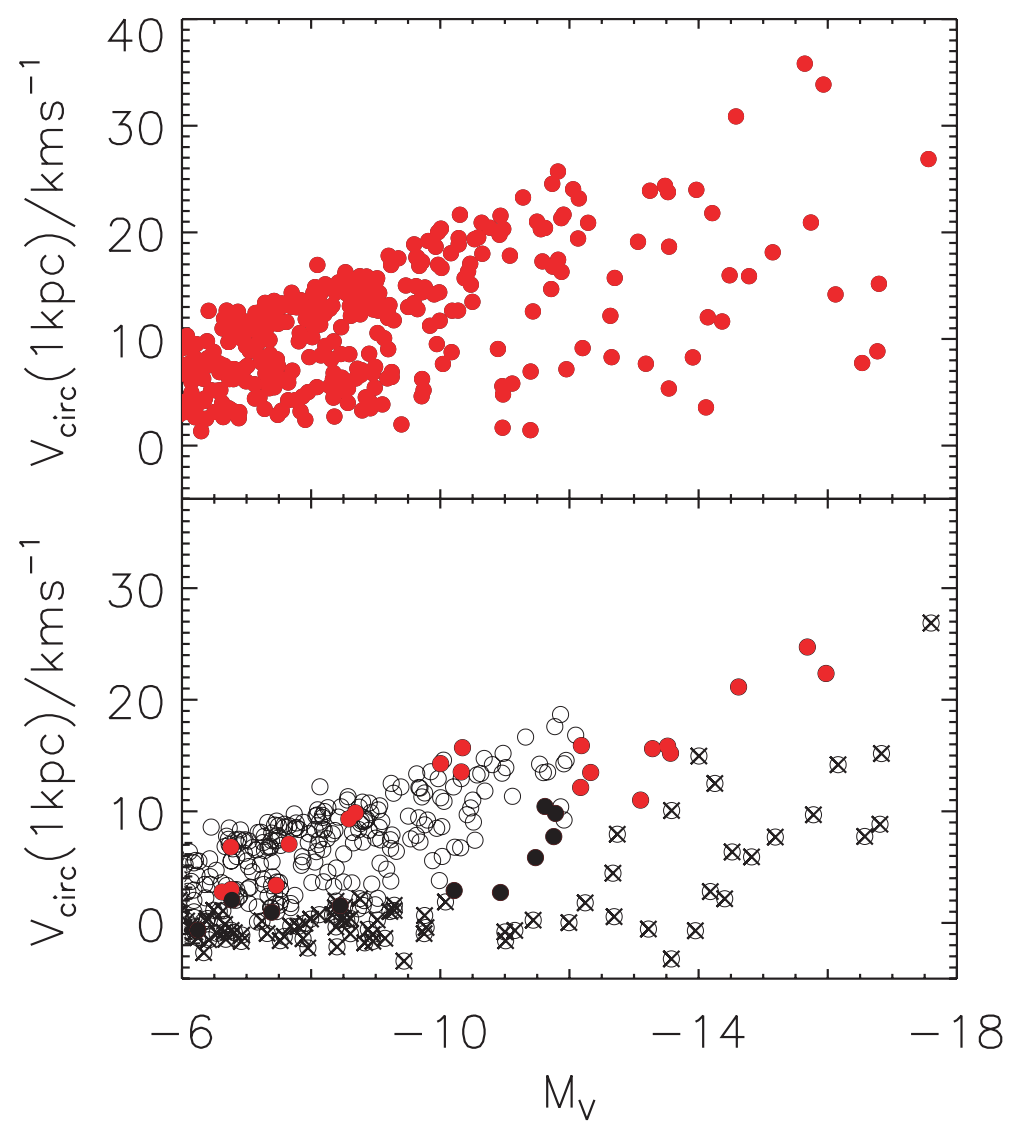

Figure 6. Plot of $v_{1 k p c}$ vs $M_{V}$ for the VL2 simulation subhaloes. In the top panel, we plot the VL2 satellites vs $M_{V}$ at $z=0$, as in B13. In the bottom panel, we represent the same VL2 satellites of the top panel with the baryonic corrections described in the text. The filled black circles represent satellites that have lost enough mass, stars are stripped and the luminosities are upper limits. Filled red circles are satellite observed at $z=0$, while circles with an $\mathrm{x}$ are subhaloes that have low probability to survive to tidal effects. Dark subhaloes are represented by empty circles.

effect producing the flattening of the cusp, and the TBTF solution is connected to the effects of SF, following episodic bursts of supernovae.

In our model, the solution to the previous quoted problems is connected to the complex interaction between DM and baryons mediated by DF. Our study is similar to those of ElZant et al. (2001, 2004), Romano-Diaz et al. (2008), Cole et al. (2011), in the sense that like them in our study DF plays an important role. However, differently from the previous studies, we consider the joint effect of several other effects (e.g., random angular momentum, angular momentum generated by tidal torques, adiabatic contraction, cooling, star formation), that in the quoted studies were not considered.

Before concluding this section, we want to recall that recently Ferrero et al. (2012) showed that the field dwarf galaxies have a similar behavior as the satellites. In order to reconcile the fact that the galaxy stellar mass function is less steep than that of dark matter halos, it is necessary to assume that the efficiency of galaxy formation decreases in a sharp fashion with decreasing halo mass. This implies that there should be a clear evidence that dwarfs having noteworthy differences in stellar mass are hosted in haloes spanning a narrow range in mass. This is not observed in their study, and moreover a large part of the galaxies 
by them studied have much less dark mass enclosed in the dwarf. One of the possibility to solve the "puzzle" is that baryonic physics, like the blow-outs produced by SF, previously discussed. However, especially in the smallest systems this solution seems very unappealing, since it is difficult to explain how baryons in a galaxy with stellar mass similar to that of globular clusters can change the inner structure of a $10^{10} M_{\odot}$ halo.

This objection moved from Ferrero et al. (2012) to the SF model, was already discussed in Sec. 3.3 of this paper. In that section, we reported that one has to expect that the $\mathrm{SF}$ mechanism becomes less effective in smaller galaxies, since in dwarfs having stellar mass $<10^{5}-10^{7} M_{\odot}$ a supernova explosion is less probable than in a larger galaxy having a larger stellar mass.

The problem envisioned by Ferrero et al. (2012) for the SF mechanism is less important in our model since the dark matter mass reduction process obtained through our model is more efficient than that connected to SF. As reported in Sec. 3.3, clumps of $1 \%$ of the halo mass can remove two times their mass, flattening cuspy profiles.

\section{Conclusions}

In the present paper, we studied the small scale problems of the $\Lambda \mathrm{CDM}$ by means of the model presented in DP09 (see also DP12a, b) following the proto-structure evolution with a semi-analytical model taking into account the effect of adiabatic contraction, dynamical friction and the exchange of angular momentum between baryons and DM, ordered and random angular momentum.

The model had already shown in DP09, DP12a,b, that the angular momentum got by the system through tidal torques and random velocities (random angular momentum), can be transferred in part to the DM from baryonic clumps of $1 \%$ the mass of the halo through $\mathrm{DF}$ action. This produces a flattening of the cusp in agreement with previous studies based on DF driven flattening of the cusp (El-Zant et al. 2001, 2004; Romano-Diaz et al. 2008) and SF driven flattening of the cusp (Navarro et al. 1996a; Gelato \& Sommersen-Larsen 1999; Read \& Gilmore 2005; Mashchenko et al. 2006, 2008).

The mass of the quoted clumps, similar to those used by El-Zant et al. (2001), and Cole et al. (2001) is quite large. In the case of the MW, using data from McMillan (2011), 1\% of the halo mass of the MW, $1.26 \pm 0.24 \times 10^{12} M_{\odot}$, corresponds to $\simeq 10^{10} M_{\odot}$ close to the total star content $\left(6.43 \pm 0.63 \times 10^{10} M_{\odot}\right)$ or the disc mass $\left(\simeq 2.4 \times 10^{10} M_{\odot}\right)$. We want to recall, that nevertheless the previous assumption, lighter clumps are more efficient. As shown by Cole et al. (2011) in their Fig. 1, the ratio among the maximum "excavated" mass to the mass of the clump increases with decreasing clump mass. While a clump of $1 \%$ of that of the halo can excavate $\simeq 2$ times its own mass, a clump with mass $0.001 \%$ of that of the halo can excavate till $\simeq 5$ times its own mass depending on the central density slope and the structure scale radius (see Eq. 11 of Cole et al. 2011).

Another assumption, used in the paper is that haloes form primarily through quiescent accretion, in agreement with the prescriptions of spherical collapse model. As previously discussed the existence of thin bulgeless disks support the previous idea, but at the same time we know that the role of mergers, in general, is important in the overall formation of structures. 
We want to stress that our results are strictly depending on the previous two ad hoc assumptions.

The MSP and TBTF problem were discussed in the same framework as follows. We studied through our model how baryons influence the inner density profile (or the inner circular velocity, $v_{1 k p c}$ ) before infall in the host halo. Then, we added the changes to $v_{1 k p c}$ after infall, produced by tidal stripping, and UV heating. We applied these corrections to the VL2 satellites, obtaining the result that their mass and number is reconciled with observations.

We also compared the distribution of spin, and the angular momentum distribution, obtained from our model, to those obtained for 14 dwarfs by VBS finding that the DM and baryons distribution is different and we did not observe the lost of angular momentum typical of many past SPH simulations.

\section{Acknowledgements}

We would like to thank the International Institute of Physics in Natal for the facilities and hospitality, Adi Zolotov, A. M. Brooks, and Charles Downing (from Exeter University) for helpful comments and for a critical reading of the paper. DCR and JCF thank CNPq and FAPES for partial financial support.

\section{References}

[1] Abadi, M.G., Navarro, J.F., Steinmetz, M., Eke, V.R. 2003, ApJ, 591, 499

[2] Anderhalden, D., Diemand, J., 2013, JCAP 08, 002

[3] Antonuccio-Delogu, V., \& Atrio-Barandela, F. 1992, ApJ, 392, 403

[4] Ascasibar Y., Yepes G., Gottlober S., 2004, MNRAS, 352, 1109

[5] Ascasibar, Y., Hoffman, Y., Gottlöber, S. 2007, MNRAS 376, 393

[6] Astashenok, A. V., and Del Popolo, A., 2012, Class. Quantum Grav. 29, 085014 (doi:10.1088/0264-9381/29/8/085014)

[7] Avila Reese V., Firmani C., Hernandez X., 1998, ApJ, 505, 37

[8] Avila Reese V., Firmani C., Klypin A., Kravtsov A., 1999, MNRAS, 310, 527

[9] V. Belokurov, V., ApJ 2006,, 647, L111-L114

[10] Bertschinger E., 1985, ApJS, 58, 39

[11] Blumenthal G. R., Faber S. M., Flores R., Primack J. R., 1986, ApJ, 301, 27

[12] Boylan-Kolchin, M., Bullock, J. S., Kaplinghat, M, 2011, MNRAS 415L, 40

[13] Boylan-Kolchin, Michael; Bullock, James S.; Kaplinghat, Manoj, 2012, MNRAS 422, 1203

[14] Brooks A. M., \& Zolotov, A., 2012, arXiv: 1207.2468

[15] Brooks, Alyson M.; Kuhlen, Michael; Zolotov, Adi; Hooper, Dan, 2013, ApJ 765, 22

[16] Buchalter, A., Jimenez, R., \& Kamionkowski, M. 2001, MNRAS, 322, 43

[17] Buchdahl, H. A., 1970, MNRAS, 150, 1-8.

[18] Bullock, J. S., Kravtsov, A. V., \& Weinberg, D. H. 2000, ApJ, 539, 517

[19] Burkert, A. 1995, ApJ, 447, L25 
[20] Cardone, V. F.; Del Popolo, A., 2012, MNRAS 427, 3176

[21] Cardone V. F., Sereno M., 2005, A\&A, 438, 545

[22] Catelan P., Theuns T., 1996, MNRAS, 282, 436

[23] Chandrasekhar, S., \& von Neumann, J. 1942, ApJ, 95, 489

[24] Chandrasekhar, S., 1943, ApJ 97, 255

[25] Colin, P., Avila-Reese, V., \& Valenzuela, O. 2000, ApJ, 542, 622

[26] Cole, D. R., Dehnen,W., \& Wilkinson, M. I. 2011, MNRAS, 416, 1118

[27] Croton D. J., et al. 2006, MNRAS, 365, 11

[28] D’Onghia, E., Burkert, A. 2004, ApJ 612,..13

[29] David J. Sand , Tommaso Treu ,1,2 Graham P. Smith, and Richard S. Ellis, The Astrophysical Journal, 604:88-107, 2004

[30] de Blok,W. J. G., Bosma, A., \& McGaugh, S. 2003, MNRAS, 340, 657

[31] de Blok, W. J. G., 2010, Advances in Astronomy Volume 2010, Article ID 789293

[32] Dekel et al., 2009, Nature 457, 451

[33] de Jong R. S., Lacey C., 2000, ApJ, 545, 781

[34] De Lucia G., Helmi A., 2008, MNRAS, 391, 14

[35] De Lucia G., Kauffmann G., Springel V., White S. D.M., Lanzoni B., Stoehr F., Tormen G., Yoshida N., 2004, MNRAS, 348, 333

[36] Del Popolo, A., Gambera, M., 1997, A\&A 321, 691

[37] Del Popolo, A., 2007, Astronomy Reports, Volume 51, 169

[38] Del Popolo, A., 2009, ApJ 698, 2093

[39] Del Popolo, A., 2011, JCAP 07, 014

[40] Del Popolo, A., 2010, MNRAS 408, 1808

[41] Del Popolo, A. \& Kroupa, P., 2009A\&A 502, 733

[42] Del Popolo, A., 2012a, MNRAS 424, 38

[43] Del Popolo, A., 2012b, MNRAS 419, 971

[44] Del Popolo, A., 2013, AIP Conference Proceedings 1548 , pp. 2-63

[45] Del Popolo, A., 2014, IJMPD 23, No. 3, 1430005

[46] Del Popolo, A., Cardone, V. F., \& Belvedere, G., 2013, MNRAS 429, 1080

[47] Del Popolo, A., Pace, F., Maydaniuk, S. P., Lima, J. A. S., Jesus, J. F., 2013, Phys. Rev D, vol. 87, Issue 4 , id. 043527

[48] Del Popolo, A., Pace, F., Lima, J. A. S., 2013a, MNRAS 430, 628

[49] Del Popolo, A., Pace, F., Lima, J. A. S., 2013b, IJMPD 22, 1350038

[50] Del Popolo, A., Hiotelis, N., 2014, JCAP 01, 047

[51] Di Cintio, A., Knebe, A., Libeskind, N. I., Brook, C., Yepes, G., Gottlöber, S., Hoffman, Y., 2013, MNRAS 431, 1220-1229

[52] Di Cintio, A., Brook, C. B., Macció, A. V., Stinson, G. S., Knebe, A., Dutton, A. A., Wadsley, J., 2014, MNRAS 437, 415

[53] Diemand, J., et al. 2008, Nature 454, 735 
[54] Diemand, J., Moore, B., \& Stadel, J. 2004, MNRAS, 353, 624

[55] Diemand, J., Kuhlen, M., Madau, P., 2007a, ApJ 657, 262

[56] Diemand, J., Kuhlen, M., and Madau, P., 2007b, ApJ 667, 859877

[57] Eisenstein D. J., Loeb A., 1995, ApJ, 439, 520

[58] Elmegreen D. M., Elmegreen B. G., Hirst A. C., 2004, ApJL, 604, L21

[59] Elmegreen D. M., Elmegreen B. G., Marcus M. T., Shahinyan K., Yau A., Petersen M., 2009, ApJ, 701, 306

[60] El-Zant A. A., Hoffman Y., Primack J., Combes F., Shlosman I., 2004, ApJ, 607, L75

[61] El-Zant, A., Shlosman, I., \& Hoffman, Y. 2001, ApJ, 560, 636

[62] Ferrero, I., Abadi, M. G., Navarro, J. F., Sales, L. V., Gurovich, S., 2012, MNRAS, 425, $2817-2823$

[63] Fall S. M., Efstathiou G., 1980,MNRAS, 193, 189

[64] Ferraro, R., 2012, AIP Conf. Proc. 1471, 103-110, arXiv:1204.6273v2

[65] Fillmore J. A., Goldreich P., 1984, ApJ, 281, 1

[66] Firmani, C., \& Avila-Reese, V. 2000, MNRAS, 315, 457

[67] Flores, R., Primack, J. R., Blumenthal, G. R., Faber, S. M., 1993, ApJ 412, 443-454

[68] Flores R. A., Primack J. R., 1994, ApJ, 427, L1

[69] Frenk, and S. D. M. White, 2008, MNRAS 391 16851711, [arXiv:0809.0898].

[70] Fukushige, T., \& Makino, J. 2001, ApJ, 557, 533

[71] Garrison-Kimmel, S., Rocha, M., Boylan-Kolchin, M., Bullock, J. S., Lally, J., 2013, MNRAS 433,3539

[72] Gelato, S., \& Sommer-Larsen, J. 1999, MNRAS, 303, 321

[73] Genzel R., et al., 2011, ApJ, 733, 101

[74] Gnedin O. Y., Kravtsov A. V., Klypin A. A., Nagai D., 2004, ApJ, 616, 16

[75] Go Ogiya, G. \& Mori, M., 2013, in preparation

[76] Goodman, J. 2000, New Astron., 5, 103

[77] Governato et al. 2010, Nature 463, 203

[78] Governato, F., Zolotov, A., Pontzen, A., Christensen, C., Oh, S. H., Brooks, A. M., Quinn, T., Shen, S., Wadsley, J., 2012, MNRAS 422, 1231

[79] Grillmair, C. J. 2006, ApJ, 645, L37

[80] Gunn J. E., 1977, ApJ, 218, 592

[81] Gunn J. E., Gott J. R., 1972, ApJ, 176, 1

[82] Gustafsson M., Fairbairn M., Sommer-Larsen J., 2006, Phys. Rev. D, 74, 123522

[83] Hiotelis, N., Del Popolo, A., 2006, Astrophysics and Space Science, 301, 167

[84] Hiotelis, N., Del Popolo, A., 2013, MNRAS 436, 163

[85] Hoffman Y., Shaham J., 1985, ApJ, 297, 16

[86] Hoyle F., 1949, in Burger J. M., van der Hulst H. C., eds, IAU and International Union of Theorethical and Applied Mechanics Symposium, Problems of Cosmological Aerodynamics. IAU, Ohio, p. 195 
[87] Hu W., Kravtsov A. V., 2003, ApJ, 584, 702

[88] Hu,W., Barkana, R., \& Gruzinov, A. 2000, Phys. Rev. Lett., 85, 1158

[89] Huss, A., Jain, B., \& Steinmetz, M. 1999a, ApJ, 517, 64

[90] Huss, A., Jain, B., \& Steinmetz, M. 1999b, MNRAS, 308, 1011

[91] Inoue, Shigeki; Saitoh, Takayuki R., 2012MNRAS.422.1902I

[92] Inoue, Shigeki; Saitoh, Takayuki R., Mon. Not. R. Astron. Soc. 418, 25272531 (2011)

[93] Irwin, M. J., et al. 2007, ApJ, 656, L13

[94] Jing, Y. P., \& Suto, Y. 2000, ApJ, 529, L69

[95] Kandrup, H. E. 1980, Phys. Rep., 63, 1

[96] Kaplinghat, M., Knox, L., \& Turner, M. S. 2000, Phys. Rev. Lett., 85, 3335

[97] Keeton C. R., 2001, ApJ, 561, 46

[98] Klypin A., Kravtsov A. V., Bullock J. S., Primack J. R., 2001, ApJ, 554, 903

[99] Klypin A., Kravtsov A. V., Valenzuela O., Prada, F., 1999, ApJ 522, 82

[100] Klypin A., Zhao H.-S., Somerville R. S., 2002, ApJ, 573, 597

[101] Komatsu, E., et al. 2009, ApJS, 180, 330

[102] Komatsu, E., Smith, K. M., Dunkley, J., et al. 2011, ApJS, 192, 18

[103] Kravtsov, A. V., Gnedin, O. Y., \& Klypin, A. A. 2004, ApJ, 609, 482

[104] Kuzio de Naray, R., Kaufmann, T., 2011, MNRAS.414.3617

[105] Lackner C. N., Ostriker J. P., 2010, ApJ, 712, 88

[106] Li, Y.-S.; De Lucia, G. Helmi, A., 2010, MNRAS 401, 2036, arXiv:0909.1291v2

[107] Le Delliou M., Henriksen R. N., 2003, A\&A, 408, 27

[108] Lukic Z., Reed D., Habib S., Heitmann K., 2009, ApJ, 692, 217

[109] Ma, C-P., Boylan-Kolchin, M., 2004, PhysRevLett 93, 021301

[110] Madau, Piero; Diemand, Jrg; Kuhlen, Michael, 2008, ApJ 679, 1260

[111] Mayer, L., Governato, F., Tobias Kaufmann, T., 2008, Adv.Sci.Lett.1:7-27

[112] Marinacci, F., Pakmor, R., and Springel, V., 2013, arXiv:1305.5360

[113] Martizzi, D., Teyssier, R., Moore, B., \& Wentz, T. 2012, MNRAS, 422, 3081

[114] Mashchenko, S., Couchman, H. M. P., \& Wadsley, J. 2006, Nature, 442, 539

[115] Mashchenko, S., Wadsley, J., \& Couchman, H. M. P. 2008, Science, 319, 174

[116] McGaugh S. S., Schombert J. M., de Blok W. J. G., Zagursky M. J., 2010, 708, L14

[117] McMillan, P. J., 2011, MNRAS 414, 2446

[118] Mestel L., 1963, MNRAS, 126, 55.

[119] Milgrom, M., 1983a, ApJ 270, 365-370

[120] Milgrom, M. 1983b, ApJ 270, 371-389

[121] Mo H. J., Mao S., White S. D. M., 1998, MNRAS, 295, 319

[122] Moore B., 1994, Nat, 370, 629

[123] Moore B., Governato F., Quinn T., Stadel J., Lake G., 1998, ApJ, 499, L5

[124] Moore, B., Quinn, T., Governato, F., Stadel, J., \& Lake, G. 1999, MNRAS, 310, 1147 
[125] Moore, B., Diemand, J., Madau, P., Zemp, M., \& Stadel, J. 2006, MNRAS, 368, 563

[126] Munshi, F., et al. 2013, ApJ 766, 56

[127] Natarajan, P. 1999, ApJ, 512, L105

[128] Navarro J. F. et al., 2010, MNRAS, 402, 21

[129] Navarro J. F., Benz W., 1991, ApJ, 380, 320

[130] Navarro J. F., Frenk C. S., White S. D. M., 1996, ApJ, 462, 563

[131] Navarro J. F., Frenk C. S., White S. D. M., 1997, ApJ, 490, 493

[132] Navarro J. F., Steinmetz M., 1997, ApJ, 478, 13

[133] Navarro J. F., Steinmetz M., 2000, ApJ, 538, 477

[134] Navarro, J. F., Eke, V. R., \& Frenk, C. S. 1996, MNRAS, 283, L72

[135] Newman A. B., Treu T., Ellis R. S., Sand D. J., Richard J., Marshall P. J., Capak P., Miyazaki S., 2009, ApJ, 706, 1078

[136] Newman A. B., Treu T., Ellis R. S., Sand D. J., 2011, ApJ, 728, 39

[137] Newman, A. B., Treu, T., Ellis, R. S., Richard, J., Sand, D. J., 2013a, ApJ 765, 25

[138] Newman, A. B., Treu, T., Ellis, R. S., Sand, D. J., Nipoti, C., Richard, J., Jullo, E., 2013b, ApJ 765,24

[139] Nipoti, C., Treu, T., Ciotti, L., Stavelli, M., 2004, MNRAS 355, 1119

[140] Noguchi M., 1998, Nat, 392, 253

[141] Noguchi M., 1999, ApJ, 514, 77

[142] Oh, S-H, C. Brook, F. Governato, E. Brinks, L. Mayer,W. J. G. de Blok ,A. Brooks, F.Walter, 2010, AJ, 142, 24

[143] Oh, S-H, de Blok, W. J. G., Brinks, E., Fabian, W., Kennicutt, R. C., Jr., 2011 AJ 141, 193

[144] Okamoto, T., Gao, L., \& Theuns, T. 2008, MNRAS, 390, 920

[145] Ostriker J. P., Steinhardt P., 2003, Science, 300, 1909

[146] Peebles P. J. E., 1969, ApJ, 155, 393

[147] Peebles P. J. E., 1980, The Large Scale Structure of the Universe. Princeton Univ. Press, Princeton, NJ

[148] Peebles, P. J. E. 2000, ApJ, 534, L127

[149] Peñarrubia et al., 2010, MNRAS 406, 1290

[150] Pontzen, Andrew; Governato, Fabio, MNRAS, Volume 421, Issue 4, pp. 3464-3471

[151] Purcell, C. W., Zentner, A. R., 2012, JCAP 12, 007

[152] Read, J. I., \& Gilmore, G. 2005, MNRAS, 356, 107

[153] Ricotti, M. 2003, MNRAS, 344, 1237

[154] Ricotti, M., \& Gnedin, N. Y. 2005, ApJ, 629, 259

[155] Ricotti, M., \& Wilkinson, M. I. 2004,MNRAS, 353, 867

[156] Ricotti, M., Pontzen, A., \& Viel, M. 2007, ApJ, 663, 53

[157] Rix H.-W., de Zeeuw P. T., Cretton N., van der Marel R. P., Carollo C. M., 1997, ApJ, 488, 702

[158] Romano-Diaz, E., Shlosman, I., Heller, C., \& Hoffman, Y. 2009, ApJ, 702, 1250 
[159] Romano-Diaz, E., Shlosman, I., Hoffman, Y., \& Heller, C. 2008, ApJ, 685, L105

[160] Romeo, A. B.,Agertz, O., Moore, B., \& Stadel, J. 2008, arXiv:astro-ph/0804.0294

[161] Ryden B. S., 1988, ApJ, 329, 589

[162] Ryden B. S., Gunn J. E., 1987, ApJ, 318, 15

[163] Sakamoto, T., \& Hasegawa, T. 2006, ApJ, 653, L29

[164] Simon, J. D.; Geha, M., 2007, AAS 211, 2602

[165] Sommer-Larsen J., Gelato S., Vedel H., 1999, ApJ, 519, 501

[166] Sommer-Larsen, J., \& Dolgov, A. 2001, ApJ, 551, 608

[167] Sommer-Larsen, J., G-otz, M., Portinari, L. 2003, ApJ, 596, 47

[168] Spergel, D. N., Verde, L., Peiris, H. V., et al. 2003, ApJS, 148, 175

[169] Springel, V., Wang, J., Vogelsberger, M., Ludlow, A., Jenkins, A., Helmi, A., Navarro, J. F., Frenk, C. S., White, S. D. M., 2008, MNRAS 391, 1685

[170] Stadel J., Potter D., Moore B., Diemand J., Madau P., Zemp M., Kuhlen M., Quilis V., 2009, MNRAS, 398, 21

[171] Starobinsky, A. A. (1980). Physics Letters B 91: 99-102

[172] Strigari, L. E., Bullock, J. S., Kaplinghat, M., Diemand, J., Kuhlen, M., Madau, P., 2007, ApJ 669,676

[173] Swaters, R. A., Madore, B. F., van den Bosch, F. C., \& Balcells, M. 2003, ApJ, 583, 732

[174] Taylor, J. E., Babul, A., 2001, ApJ 559:716-735

[175] Taylor, J. E., Silk, J., \& Babul, A. 2004, in IAU Symp. 220, Dark Matter in Galaxies, ed. S.

D. Ryder et al. (San Francisco, CA: ASP), 91

[176] Toth, G., \& Ostriker, J. P. 1992, ApJ, 389, 5

[177] Treu T., Koopmans L. V. E., 2002, ApJ, 575, 87

[178] van den Bergh S., Abraham R. G., Ellis R. S., Tanvir N. R., Santiago B. X., Glazebrook K. G., 1996, AJ, 112, 359

[179] van den Bosch F. C., 1998, ApJ, 507, 601

[180] van den Bosch, F. C., \& Swaters, R. A. 2001,MNRAS, 325, 1017 (VS)

[181] van den Bosch, F. C., Abel, T., Croft, R. A. C., Hernquist, L., White, S. D. M., 2002, ApJ 576, 21, astro-ph/0201095

[182] van den Bosch F. C., Burkert A., Swaters R. A., 2001, MNRAS, 326, 1205 (VBS)

[183] van den Bosch, F. C., Robertson, B. E., Dalcanton, J. J., \& de Blok,W. J. G. 2000, AJ, 119, 1579

[184] van den Bosch, F. C., 2002, MNRAS, 332, 456

[185] Vera-Ciro, C. A., Helmi, A. Starkenburg, E., Breddels, M. A., 2013, MNRAS 428, 1696

[186] Vitvitska M., Klypin A. A., Kravtsov A. V., Wechsler R. H., Primack J. R., Bullock J. S., 2002, ApJ, 581, 799

[187] Wang, J., Frenk, C. S., Navarro, J. F., Gao, L., Sawala, T., 2012, MNRAS 424, 2715

[188] Weinberg, S., Rev. Mod. Phys., 1989, 61, 1

[189] White M., 2001, A\&A, 367, 27

[190] White S. D. M., 1984, ApJ, 286, 38 
[191] White S. D. M., Frenk C. S., 1991, ApJ, 379, 52

[192] White S. D. M., Rees M. J., 1978, MNRAS, 183, 341

[193] White S. D. M., Zaritsky D., 1992, ApJ, 394, 1

[194] Williams, L. L. R., Babul, A., \& Dalcanton, J. J. 2004, ApJ, 604, 18

[195] Willman, B., et al. 2005a, ApJ, 626, L85

[196] Yang-Shyang Li, Gabriella De Lucia and Amina Helmi1 arXiv: 0909.1291v2

[197] Zaroubi, S., Naim, A., \& Hoffman, Y. 1996, ApJ, 457, 50

[198] Zentner, A. R., \& Bullock, J. S. 2003, ApJ, 598, 49

[199] Zolotov, A., Brooks, A. M., Willman, B., Governato, F., Pontzen, A., Christensen, C., Dekel,

A., Quinn, T., Shen, S., Wadsley, J., 2012, ApJ 761, 71

[200] Zucker, D. B., et al. 2006a, ApJ, 643, L103 